\title{
Extended massive gravity in three dimensions
}

\author{
Hamid R. Afshar, Eric A. Bergshoeff and Wout Merbis \\ Centre for Theoretical Physics, University of Groningen, \\ Nijenborgh 4, 9747 AG Groningen, The Netherlands \\ E-mail: h.r.afshar@rug.nl, E.A.Bergshoeff@rug.nl, w.merbis@rug.nl
}

\begin{abstract}
Using a first order Chern-Simons-like formulation of gravity we systematically construct higher-derivative extensions of general relativity in three dimensions. The construction ensures that the resulting higher-derivative gravity theories are free of scalar ghosts. We canonically analyze these theories and construct the gauge generators and the boundary central charges. The models we construct are all consistent with a holographic $c$-theorem which, however, does not imply that they are unitary. We find that Born-Infeld gravity in three dimensions is contained within these models as a subclass.
\end{abstract}

Keywords: AdS-CFT Correspondence, Chern-Simons Theories, Classical Theories of Gravity

ARXIV EPRINT: 1405.6213 


\section{Contents}

1 Introduction 1

2 Extended massive gravity models $\quad 5$

2.1 Action principle $\quad 7$

2.2 Absence of scalar ghosts 8

3 Extended new massive gravity $\quad 9$

$\begin{array}{lll}3.1 & \text { Linearization } & 11\end{array}$

$\begin{array}{ll}3.2 & \text { Critical lines and the tricritical point } \\ \end{array}$

4 Anti-de Sitter holography $\quad 15$

$\begin{array}{ll}\text { 4.1 Gauge symmetries in CS-like theories } & 16\end{array}$

$\begin{array}{lll}4.2 & \text { AdS boundary conditions and the central charge } & 16\end{array}$

$\begin{array}{lll}4.3 & \text { Logarithmic anomalies } & 18\end{array}$

$\begin{array}{ll}4.4 \text { Holographic } c \text {-theorem } & 20\end{array}$

5 Discussion $\quad 22$

A Charges in Chern-Simons-like theories $\quad \mathbf{2 5}$

$\begin{array}{lll}\text { A.1 Canonical analysis } & 25\end{array}$

$\begin{array}{lll}\text { A.2 } & \text { First class constraint functions } & 27\end{array}$

$\begin{array}{lll}\text { A.3 Boundary charges } & 28\end{array}$

B Extended gravitational Chern-Simons term $\quad 29$

$\begin{array}{lll}\text { B.1 Linearization } & 30\end{array}$

B.2 Central charge 31

\section{Introduction}

Three-dimensional gravity models are an interesting playground to study problems in quantum gravity. In particular, three dimensional general relativity (GR) with or without cosmological constant is known to be described by a Chern-Simons (CS) gauge theory $[1,2]$, at least classically. The presence of a negative cosmological constant not only makes it possible to have black hole solutions $[3,4]$ but also leads to the boundary global degrees of freedom by defining an asymptotic boundary and imposing appropriate boundary conditions [5]. In spacetimes with asymptotic boundaries, one can define the asymptotic symmetry group as the group of boundary condition preserving gauge transformations. For asymptotically locally anti-de Sitter $\left(\mathrm{AdS}_{3}\right)$ spacetimes, the asymptotic symmetry group consists of two copies of the Virasoro algebra with a classical central extension. This approach to quantum 
gravity is one of the earliest applications of the AdS/CFT correspondence which organizes our understanding of a quantum gravity theory in terms of a dual conformal field theory (CFT) and vice versa.

Although the lack of local degrees of freedom in three dimensional GR can be regarded as a technical simplification, it makes it less interesting from a perturbative field theoretic point of view in which propagating degrees of freedom play an important role. One way to compensate for this shortcoming in a purely gravitational manner is to add higherderivative terms to GR, which can lead to new massive spin-2 modes in the spectrum $[6,7]$. From the CFT point of view this would correspond to deforming the dual CFT by a new operator which couples to this new massive bulk mode. Deformations in unitary two dimensional CFTs are restricted by Zamolodchikov's $c$-theorem [8]. The presence of a holographic $c$-theorem [9] can clarify the role of the higher-curvature terms [10], as it implies a restriction on the coupling constants of these higher-derivative interactions in the bulk.

The main aim of this paper is to systematically construct higher-derivative extensions of three dimensional gravity which are free of scalar ghosts and consistent with a holographic $c$-theorem. In this construction, we exploit a first order formulation which is denoted as the 'Chern-Simons-like' formulation [11, 12]. In this formulation the dynamical fields are a set of one-forms, denoted by flavor indices $r, s, t, \ldots$, taking values in the three-dimensional Lorentz group $\mathrm{SO}(2,1)$,

$$
a^{r}=\left(a_{\mu}^{r a} d x^{\mu}\right) J_{a}, \quad J_{a} \in s o(2,1) .
$$

The CS-like Lagrangian three-form constructible from these Lorentz-valued one-forms can be defined as

$$
L_{\mathrm{CS}-\mathrm{like}}=\frac{1}{2}\left\langle g_{r s} a^{r} \wedge d a^{s}+\frac{2}{3} f_{r s t} a^{r} \wedge a^{s} \wedge a^{t}\right\rangle .
$$

Here $g_{r s}$ is a symmetric and invertible metric on the flavor space, and the coupling constants $f_{r s t}$ define a totally symmetric flavor tensor. This construction is completely gauge invariant under $\mathrm{SO}(2,1)$ once we use the spin-connection $\omega$ as the gauge field and the trace over Lorentz indices in the three dimensional representation of $\operatorname{SO}(2,1)$ [13]. The corresponding bilinear form, structure constants and covariant derivative are given by

$$
\left\langle J_{a}, J_{b}\right\rangle=\eta_{a b}, \quad\left[J_{a}, J_{b}\right]=\epsilon_{a b}^{c} J_{c} \quad \text { and } \quad \mathcal{D} \equiv d+\frac{1}{2}[\omega,] .
$$

The dualized curvature two-form is then given by ${ }^{1}$

$$
R^{a}=\mathcal{D} \omega^{a}=d \omega^{a}+\frac{1}{2} \epsilon_{b c}^{a} \omega^{b} \omega^{c} .
$$

Whenever the combinations $\epsilon_{b c}^{a} f^{r}$ st are the structure constants of some Lie algebra and $g_{r s} \eta_{a b}$ a bilinear form on this algebra, then the theory defined by (1.2) is actually a ChernSimons gauge theory. There are two parity preserving ${ }^{2}$ gravity models in three dimensions

\footnotetext{
${ }^{1}$ Unless stated explicitly, we normally use the notation in which wedge products are implicit.

${ }^{2}$ Here, by 'parity preserving' theories we mean those Lagrangians which have a definite parity, $P L= \pm L$, while 'parity violating' Lagrangians have no definite parity.
} 
for which this is the case. They are Einstein gravity, of even parity, and conformal gravity ${ }^{3}$ which is odd under parity. They have the following first order actions:

$$
\begin{aligned}
& \text { Parity-even, } \quad S_{\text {Einstein }} \equiv S_{0}=-\frac{1}{\kappa^{2}} \int\left\langle e \wedge\left(R-\frac{\Lambda_{0}}{3} e \wedge e\right)\right\rangle, \\
& \text { Parity-odd, } \quad S_{\text {Conformal }} \equiv S_{1}=\frac{1}{2 \kappa^{2} \mu} \int\left\langle\omega \wedge\left(d \omega+\frac{2}{3} \omega \wedge \omega\right)+2 f \wedge \mathcal{D} e\right\rangle .
\end{aligned}
$$

Here $\kappa^{2}=8 \pi G$ is the three dimensional Planck length and $\Lambda_{0}$ the cosmological constant with the dimension of (mass) $)^{2}$ while $\mu$ is a parameter with the dimension of mass. This amounts to a dimensionless coupling constant for conformal gravity which is a conformally invariant theory. Due to the lack of any local degrees of freedom, these models can be written purely as Chern-Simons gauge theories for $\mathrm{SO}(2,2)$ and $\mathrm{SO}(2,3)$ respectively, where $e, \omega$ and $f$ correspond to the gauge fields for translations, rotations and special conformal transformations, respectively [1, 2, 16]. For a recent discussion of these theories, see [17].

In this work we will consider extensions of the above theories in the CS-like formulation to include dynamical spin-2 degrees of freedom by introducing sufficiently many auxiliary one-forms in a parity preserving way. After integrating out these auxiliary one-forms, the resulting theory is a parity-even or a parity-odd higher-derivative theory of gravity. The most general set of parity violating models can be constructed by combining the parityeven and odd theories. The first of these parity violating models is topologically massive gravity (TMG) [6], which is the sum of the actions (1.5) and (1.6) and propagates a single massive helicity- 2 state with one local degree of freedom. An example of a parity preserving extension which describes two helicity- \pm 2 states, with two degrees of freedom, is 'new massive gravity' (NMG) [7, 18].

Both TMG and NMG may be described in terms of a first order CS-like formulation [11, 19-21]. The first order formulation of conformal gravity given in (1.6), which is a threederivative action in terms of the metric is an example of how one can exchange a higherderivative action for a first order action containing auxiliary fields. In the case of conformal gravity this auxiliary field is the one-form $f^{a}[14,15]$ — see [22] for a recent review. In $[11,21]$ this approach was extended to four-derivative actions by introducing two extra auxiliary one-form fields $\left(f^{a}, h^{a}\right)$ to obtain NMG, which for future reference we denote by $S_{2}$ :

$$
S_{\mathrm{NMG}} \equiv S_{2}=S_{0}-\frac{1}{\kappa^{2} m^{2}} \int\langle f \wedge(R+e \wedge f)+h \wedge \mathcal{D} e\rangle
$$

The equivalent four derivative action is recovered after integrating out the two auxiliary fields.

Originally, NMG was not found in the first order form given above. Instead, it was constructed by extending GR in 3D with higher-curvature $R_{\mu \nu} R^{\mu \nu}$ and $R^{2}$ terms. It was found that the theory describes the two degrees of freedom of a massive spin- 2 degree of freedom only for a particular combination of higher-curvature terms [7, 18, 23]. Moving away from this special combination introduces a third scalar degree of freedom corresponding to a Boulware-Deser ghost mode [24]. Interestingly, it is only possible to write down

\footnotetext{
${ }^{3}$ This model is sometimes referred to as conformal Chern-Simons gravity, denoted as CSG [14, 15].
} 
a CS-like formulation of $R^{2}$ extended gravity if the higher-curvature terms occur precisely in the ghost-free NMG combination. We will use this observation as a guiding principle to construct further generalizations of higher-derivative massive gravity in three dimensions which, as we will show explicitly, are free of scalar ghost excitations.

For the parity-even sector, we start by considering Einstein gravity (1.5). First, we include two auxiliary fields to obtain NMG. Next, we will show that by adding an additional set of two auxiliary fields we obtain a six derivative theory which generically propagates two massive spin-2 modes. The resulting theory is a combination of the $R^{3}$ terms considered in [25] as extended NMG and $R \square R$ terms also considered in [26] as parity-even tricritical (PET) gravity. We analyze the linear spectrum of the theory and find that even though for a general choice of parameters the scalar ghosts are absent, one of the two massive spin-2 modes is either tachyonic or a ghost. An exception is the cubic extended NMG model in which the $R \square R$ terms are absent [25] and only one massive spin-2 mode is propagated. Upon assigning a wrong sign to the EH term, this single massive spin-2 mode is not a ghost. All higher curvature extended models of NMG, however suffer from a unitarity conflict in AdS, as their corresponding boundary central charge is negative. These results imply that even though the scalar ghosts can be avoided in higher-derivative gravity theories, the unitarity problems persist, either in the form of massive spin-2 ghosts or negative boundary central charge.

These problems become less urgent at special points in the parameter space where either one or both of the massive modes become massless and a degeneracy takes place. At these points the dual CFT obtains a non-diagonalizable Jordan cell [27] and after adopting the appropriate logarithmic boundary conditions [28-30] becomes a logarithmic CFT (LCFT) - see [31] for a recent review. LCFTs are known to be non-unitary, however, they have a range of applications in condensed matter physics and statistical mechanics.

In the parity-odd sector, we start with conformal gravity (1.6). In this case, adding two auxiliary fields will break the conformal symmetry of the original theory, and the resulting five derivative theory propagates three local degrees of freedom: a 'partially massless' mode [32] and the two helicity- \pm 2 states of a massive spin- 2 mode. In this case, there is no way to tune the mass of the massive mode to zero. However, there is a special point where the massive mode degenerates with the partially massless mode.

In both sectors, all auxiliary fields and consequently all higher-derivative terms in the action are engendered by the Schouten and Cotton tensors, defined below in (2.3) and (2.5). The novelty, due to the presence of the Cotton tensor, is that it allows for actions containing terms with derivatives of curvatures consistent with a holographic $c$-theorem. In this way the class of higher-derivative theories admitting a holographic $c$-theorem in three dimensions is larger than the class of theories considered in [25, 33], which only included higher-curvature terms containing Schouten tensor. In fact we prove that the absence of the scalar ghosts imply an underlying holographic $c$-theorem in the bulk. ${ }^{4}$ Furthermore, since the resulting higher-derivative gravity theory contains massive spin- 2 ghosts, this shows that the presence of such a holographic $c$-theorem does not guarantee unitarity in the bulk theory.

\footnotetext{
${ }^{4}$ For a similar conclusion about higher curvature extensions of NMG see [34].
} 
This paper is organized as follows. In section 2 we describe the general procedure to construct higher-derivative gravities in the CS-like formulation. We derive a general action principle for the parity-even theories and the parity-odd theories separately and show that all of these extensions contain the required secondary (and second-class) constraints needed to remove the Boulware-Deser ghost. In section 3 we explicitly construct the six and eight derivative extension of GR and analyze the linear spectrum of the former to verify that it propagates two massive spin-2 excitations. By explicitly deriving the kinetic and the mass terms of the bulk modes in the Lagrangian, we show that one of the two massive spin-2 modes is either tachyonic or a ghost. There are, however, critical lines and points in the parameter space where the massive modes either disappear or become massless and degenerate with the pure gauge mode. In section 4 we discuss AdS holography for these models. We identify the conserved boundary charges and show that, when adopting Brown-Henneaux boundary conditions, the asymptotic symmetry algebra consists of two copies of the Virasoro algebra. We compute the semi-classical central charge for the six derivative model and give an expression for the new anomalies when the central charge becomes zero. The dual CFT at these points is expected to be logarithmic as they lead to the appearance of Jordan cells. Furthermore, we show the consistency of all the theories constructed in this way with a holographic c-theorem. Finally, we have included two appendices. In appendix A we discuss the identification of the first class constraints of the CS-like theories which generate the gauge symmetries and the corresponding boundary charges. Appendix B is devoted to the analysis of the parity-odd five-derivative extension of the gravitational Chern-Simons term.

\section{$2 \quad$ Extended massive gravity models}

In this section, we give a procedure to derive higher-derivative extensions of 3D GR which propagate multiple massive spin-2 particles. The extensions are obtained from an auxiliary field formalism which, as we will show, guarantees the freedom from scalar ghosts. However, as we will show in section 3.1, the higher-derivative nature of the theory does lead to the presence of massive spin- 2 ghosts.

Our starting point is a first order, Chern-Simons-like formalism [11, 12], defined by a Lagrangian three-form depending on the dreibein $e^{a}$, the dualized spin-connection $\omega^{a}$ and a number of new auxiliary Lorentz vector valued one-forms $f_{I}^{a}$ and $h_{I}^{a}$. The advantage of this approach is that it automatically leads to higher-derivative terms which are free of scalar ghosts, as we will show below.

The construction is such that the field equations will always ensure the vanishing of the torsion two-form

$$
T^{a}=\mathcal{D} e^{a}=d e^{a}+\epsilon^{a}{ }_{b c} \omega^{b} e^{c}=0 .
$$

Assuming the invertibility of the dreibein it is possible to solve this equation for the spinconnection in terms of the dreibein: $\omega^{a}=\omega^{a}(e)$. Varying the Einstein gravity action (1.5) w.r.t. $e^{a}$ gives the equation $R^{a}=\frac{1}{2} \Lambda_{0} \epsilon^{a b c} e_{b} e_{c}$, which can be written in the metric form as $G_{\mu \nu}+\Lambda_{0} g_{\mu \nu}=0$, where $G_{\mu \nu}=R_{\mu \nu}-\frac{1}{2} R g_{\mu \nu}$ is the Einstein tensor. 
Varying the conformal gravity action (1.6) w.r.t. $f^{a}, \omega^{a}$ and $e^{a}$ gives the field equations,

$$
\begin{aligned}
& \mathcal{D} e^{a}=0, \\
& R^{a}+\epsilon^{a}{ }_{b c} f^{b} e^{c}=0, \\
& \mathcal{D} f^{a}=0
\end{aligned}
$$

Assuming the invertibility of the dreibein, the auxiliary field $f^{a}$ can be solved for in terms of the curvature two-form as

$$
f_{\mu \nu} \equiv f_{\mu}^{a} e_{\nu a}=-\left(R_{\mu \nu}-\frac{1}{4} R g_{\mu \nu}\right) \equiv-S_{\mu \nu}(e) .
$$

The last equation in (2.2) then gives a third order differential equation for the dreibein: $C_{\mu \nu}(e) \equiv e^{-1} \epsilon_{(\mu \mid}^{\alpha \beta} \nabla_{\alpha} S_{\beta \mid \nu)}=0$. Here $S_{\mu \nu}$ and $C_{\mu \nu}$ are the symmetric Schouten and Cotton tensors respectively, constructed from the dreibein $e^{a}$.

Varying the NMG action (1.7) the following field equations arise:

$$
\begin{aligned}
& \mathcal{D} e^{a}=0, \\
& R^{a}+\epsilon^{a b c} e_{b} f_{c}=0, \\
& \mathcal{D} f^{a}+\epsilon^{a b c} e_{b} h_{c}=0, \\
& \mathcal{D} h^{a}+\frac{1}{2} \epsilon^{a b c}\left(f_{b} f_{c}-2 m^{2} e_{b} f_{c}-m^{2} \Lambda_{0} e_{b} e_{c}\right)=0 .
\end{aligned}
$$

The first equation in (2.4) is the torsion constraint, the second one is solved as in (2.3) and the third equation gives,

$$
h_{\mu \nu} \equiv h_{\mu}{ }^{a} e_{\nu a}=e^{-1} \epsilon_{(\mu \mid}^{\alpha \beta} \nabla_{\alpha} S_{\beta \mid \nu)}=C_{\mu \nu}(e) .
$$

The last equation in (2.4) then leads to an equation for the dreibein which is fourth order in derivatives.

Looking at equations (2.2) and (2.4) suggests to continue this logic to obtain arbitrarily higher-derivative extensions of 3D GR. Inspired by the above we consider the following schematic extension of the equations:

$$
\begin{array}{cl}
1 & \mathcal{D} e^{a}=0, \\
2 & R^{a}+\epsilon^{a b c} e_{b} f_{1 c}=0, \\
3 & \mathcal{D} f_{1}^{a}+\epsilon^{a b c} e_{b} h_{1 c}=0, \\
4 & \mathcal{D} h_{1}{ }^{a}+\epsilon^{a b c} e_{b} f_{2 c}+\ldots=0, \\
\vdots & \vdots \\
2 N+1 & \mathcal{D} f_{N}{ }^{a}+\epsilon^{a b c} e_{b} h_{N c}+\ldots=0, \\
2 N+2 & \mathcal{D} h_{N}{ }^{a}+\ldots=0 .
\end{array}
$$

The structure of these equations is such that they may be solved one after the other, starting with the first one, in terms of derivatives acting on the dreibein. The number appearing before each equation denotes the maximum number of derivatives of the dreibein which 
may appear in the equation after all fields have been solved. The dots denote terms which may contain fewer derivatives or an equal number of derivatives acting on $e^{a}$.

The first equation in (2.6) solves for the spin-connection in terms of the dreibein. The next two equations are already solved as in (2.3) and (2.5). The other auxiliary form fields $\left(f_{I}, h_{I}\right)$ can be obtained in terms of $e$ and derivatives acting on it, such that the final equation is a higher-derivative field equation for the dreibein. This set of equations may terminate with an equation for $\mathcal{D} h_{N}$ or $\mathcal{D} f_{N+1}$. The final equation then becomes, an evenor an odd-order partial differential equation for the dreibein corresponding to a parity-even or parity-odd theory respectively.

We can diagramatise the even and odd cases as follows
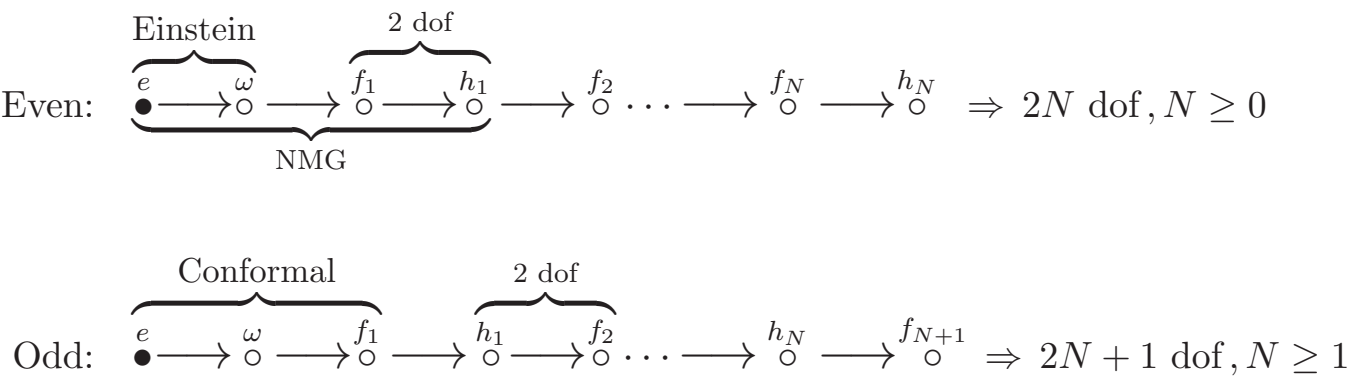

The sequential form of the diagram shows which fields are solved in terms of which ones. The filled circle denotes the assumption of invertibility of the dreibein. All other fields (open circles) need not be invertible.

\subsection{Action principle}

In both even and odd cases the set of equations (2.6) can be integrated to an action by the same general procedure. The field with the highest number of derivatives on the dreibein ( $h_{N}$ for even parity, $f_{N+1}$ for odd parity) can be used as a multiplier for the torsion constraint. The field with one derivative less will be used to multiply the second equation, and so on, until half of the field equations have been used. The rest of the field equations then follow from the action by varying the fields with a lower number of derivatives acting on the dreibein. This procedure guarantees that the highest number of derivatives appearing in the action after solving for all the auxiliary fields is $2 N+2$ for the parity-even models and $2 N+3$ for the parity-odd models. We find the following actions for the parity-even and parity-odd cases.

Parity-even models. The parity preserving extensions of the Einstein gravity action (1.5) in CS-like form can be obtained from the following recursive action:

$$
\begin{gathered}
S_{2 N}=S_{2 N-2}+\frac{\kappa^{-2}}{\left(m^{2}\right)^{N}} \int\left[\sum_{I+J=N}\left\langle f_{I} \wedge \mathcal{D} h_{J}\right\rangle+\sum_{I+J+K=N+1} \alpha_{I J K}\left\langle f_{I} \wedge f_{J} \wedge f_{K}\right\rangle\right. \\
\left.+\sum_{\substack{I+J+K=N \\
J, K \neq 0}} \beta_{I J K}\left\langle f_{I} \wedge h_{J} \wedge h_{K}\right\rangle\right]
\end{gathered}
$$


where $I=0,1, \cdots, N$ in both $f_{I}$ and $h_{I}$ with $\left(f_{0}, h_{0}\right) \equiv(e, \omega)$. The starting value in this recursive relation is given by the Einstein gravity action $S_{0}$ given in eq. (1.5). As an example, the action $S_{2}$ is already constructed in eq. (1.7).

Parity-odd models. The parity preserving extension of (1.6) in CS-like form can be obtained from the following recursive action:

$$
\begin{aligned}
S_{2 N+1}=S_{2 N-1} & +\frac{\kappa^{-2}}{\mu\left(\mu^{2}\right)^{N}} \int\left[\sum_{I+J=N}\left\langle h_{I} \wedge \mathcal{D} h_{J}\right\rangle+\sum_{\substack{I+J+K=N \\
I, J, K \neq 0}} \alpha_{I J K}\left\langle h_{I} \wedge h_{J} \wedge h_{K}\right\rangle\right. \\
& \left.+\sum_{I+J=N+1}\left\langle f_{I} \wedge \mathcal{D} f_{J}\right\rangle+\sum_{\substack{I+J+K=N+1 \\
K \neq 0}} \beta_{I J K}\left\langle f_{I} \wedge f_{J} \wedge h_{K}\right\rangle\right] .
\end{aligned}
$$

Here $I=0,1, \cdots, N+1$ in $f_{I}$ and $I=0,1, \cdots, N$ in $h_{I}$ with $\left(f_{0}, h_{0}\right) \equiv(e, \omega)$. The starting value in this recursive relation is given by the conformal gravity action $S_{1}$ given in eq. (1.6). As an example we give here the explicit form of the next action $S_{3}$ :

$$
S_{3}=S_{1}+\frac{1}{\kappa^{2} \mu^{3}} \int\left\langle e \wedge \mathcal{D} f_{2}+h_{1} \wedge\left(R+e \wedge f_{1}\right)+\alpha f_{1} \wedge \mathcal{D} f_{1}\right\rangle
$$

We will analyze this model in appendix B.

Not all couplings $\alpha_{I J K}$ and $\beta_{I J K}$ in (2.7) and (2.8) are physical. For a given $N$ we have $2 N$ auxiliary fields in the even sector and $2 N+1$ in the odd case which can be rescaled such that the same number of coefficients may be set to unity. In eqs. (2.7) and (2.8) we have already exhausted $N+1$ and $N+2$ of these rescalings, respectively, by canonically normalizing the $N+1$ and $N+2$ kinetic terms. Similarly, we also have the freedom to redefine the auxiliary fields as $f_{N} \rightarrow f_{N}+a m^{2} f_{N-1}+\ldots$ for some arbitrary constant $a$ (and likewise for $\left.h_{N}\right)$. Such field redefinitions can always be used to simplify or cancel terms appearing in $S_{2 N}$ and $S_{2 N+1}$. In the concrete examples presented in eqs. (1.7) and (2.9), and the ones coming later, we have used such shifts to cancel the kinetic terms of the lower order action.

In this work we will only analyze extensions which preserve parity. It is straightforward to extend the analysis to parity-violating models, such as TMG, by taking the sum of an even and odd parity theory. We will explicitly construct the even and odd parity extensions up to eight derivatives in the metric formalism. In section 3.1, we will perform the linearized analysis and confirm that generically each set of auxiliary fields will add 2 massive spin2 degrees of freedom. Before closing this section, we will comment on the absence of scalar ghosts and the growth of local degrees of freedom by adding each $(f, h)$-pair of auxiliary fields.

\subsection{Absence of scalar ghosts}

The advantage of the first order formulation over the metric form is that it is relatively easy to count the number of local degrees of freedom (dof) and identify the second class constraints which remove the Boulware-Deser scalar ghost. They arise from the symmetry 
of the auxiliary fields $h_{\mu \nu}$ and $f_{\mu \nu}$,

$$
f_{[\mu \nu]}=0, \quad h_{[\mu \nu]}=0 .
$$

These constraints can be derived directly from the equations of motion (2.6) by acting on them with an exterior derivative and using that $d^{2}=0$. By invertibility of the dreibein, the first equation in (2.6) simplifies to $f_{1 a} e^{a}=0$ and the second one gives $h_{1 a} e^{a}=0$, whose spatial projections are secondary constraints in a Hamiltonian formulation of the theory [11, 12]. The counting of degrees of freedom was shown in [11] for NMG but it can be generalized to all CS-like theories considered in the preceding section. The absence of additional scalar degrees of freedom then follows from a counting similar to the NMG case, provided that the secondary constraints (2.10) are second class and do not lead to further tertiary constraints. ${ }^{5}$ After a space-time decomposition of the fields, the time components $f_{0}^{a}$ and $h_{0}^{a}$, become Lagrange multipliers for a set of six primary constraints and the spatial components of the fields, $f_{i}^{a}$ and $h_{i}^{a}$, add to the canonical variables of the theory. Along with the additional secondary constraints $f_{[i j]}^{a}=0$ and $h_{[i j]}^{a}=0$, each pair of auxiliary fields will add

$$
\frac{1}{2}(12-6-2)=2
$$

degrees of freedom to the theory. These two degrees of freedom correspond to the two helicity states of a massive spin-2 mode in three dimensions. This counting works for all vector valued one-form pairs $\left(f_{I}, h_{J}\right)$. Hence, any action which gives the equations of motion with the general structure given in eq. (2.6) is guaranteed to produce a higherderivative extension of gravity in three dimensions, free of scalar ghosts.

\section{$3 \quad$ Extended new massive gravity}

In this section we construct the extensions in the parity-even sector given in eq. (2.7) up to eight derivatives. A similar analysis for the odd case is done in appendix B up to seven derivatives. Below we will first introduce the model. Next, in section 3.1 we will perform a linearized analysis of the model while in section 3.2 we will investigate critical points and critical lines in the parameter space.

The NMG action $S_{2}$ which is fourth order in derivatives was already given in eq. (1.7). The next step is to construct the six derivative action $S_{4}$. Its Lagrangian three-form ${ }^{6}$ can be derived using the recursive action (2.7). We find the following result:

$$
\begin{aligned}
L_{4}=-e_{a} & \left(\sigma R^{a}-\frac{\Lambda_{0}}{6} \epsilon_{a b c} e^{b} e^{c}\right)+\frac{1}{2 m^{2}} \epsilon_{a b c} e^{a} f_{1}{ }^{b} f_{1}{ }^{c}-\frac{1}{m^{4}}\left[e_{a} \mathcal{D} h_{2}{ }^{a}\right. \\
& \left.+\frac{a}{6} \epsilon_{a b c} f_{1}{ }^{a} f_{1}{ }^{b} f_{1}{ }^{c}+f_{2 a}\left(R^{a}+\epsilon^{a b c} e_{b} f_{1 c}\right)+b h_{1 a}\left(\mathcal{D} f_{1}{ }^{a}+\frac{1}{2} \epsilon^{a b c} e_{b} h_{1 c}\right)\right],
\end{aligned}
$$

\footnotetext{
${ }^{5}$ In this counting we assume that adding these new auxiliary fields does not change the number of gauge symmetries. This is actually what happens for conformal gravity; the presence of additional symmetries cancels the degree of freedom introduced by $f^{a}$, see [15].

${ }^{6}$ We define the Lagrangian three form $L$ and the Lagrangian density $\mathcal{L}$ as $S=\frac{1}{\kappa^{2}} \int L=\frac{1}{\kappa^{2}} \int e d^{3} x \mathcal{L}$, where $e$ denotes the determinant of the dreibein.
} 
where we have introduced a sign parameter $\sigma= \pm 1$ and two arbitrary dimensionless parameters $a, b$. The dimensionful parameters $\Lambda_{0}$ and $m^{2}$ were already introduced in eqs. (1.5) and (1.7).

The equations of motions for this Lagrangian, obtained by varying with respect to $h_{2}{ }^{a}$, $f_{2}{ }^{a}, h_{1}{ }^{a}, f_{1}{ }^{a}, \omega^{a}$ and $e^{a}$, respectively, are given by

$$
\begin{aligned}
& \mathcal{D} e^{a}=0, \\
& R^{a}+\epsilon^{a b c} e_{b} f_{1 c}=0, \\
& \mathcal{D} f_{1}{ }^{a}+\epsilon^{a b c} e_{b} h_{1 c}=0, \\
& b \mathcal{D} h_{1}{ }^{a}+\frac{1}{2} \epsilon^{a b c}\left(a f_{1 b} f_{1 c}+2 e_{b} f_{2 c}-2 m^{2} e_{b} f_{1 c}\right)=0, \\
& \mathcal{D} f_{2}{ }^{a}+\epsilon^{a b c}\left(b f_{1 b} h_{1 c}+e_{b} h_{2 c}\right)=0, \\
& \mathcal{D} h_{2}{ }^{a}+\frac{1}{2} \epsilon^{a b c}\left(2 f_{2 b} f_{1 c}+b h_{1 b} h_{1 c}-m^{2} f_{1 b} f_{1 c}-\Lambda_{0} m^{4} e_{b} e_{c}-2 m^{4} \sigma e_{b} f_{1 c}\right)=0 .
\end{aligned}
$$

The first equation imposes the zero torsion constraint and allows the spin-connection to be solved for in terms of the dreibein. Moving down the line, we find for the fields $f_{1 \mu \nu}$ and $h_{1 \mu \nu}$ the following expressions:

$$
f_{1 \mu \nu}=-S_{\mu \nu}(e) \quad \text { and } \quad h_{1 \mu \nu}=C_{\mu \nu}(e) .
$$

These in turn fix the expressions for $f_{2 \mu \nu}$ and $h_{2 \mu \nu}$ as follows:

$$
\begin{aligned}
& f_{2 \mu \nu}=-b D_{\mu \nu}+a\left(P_{\mu \nu}-\frac{1}{4} P g_{\mu \nu}\right)-m^{2} S_{\mu \nu}, \\
& h_{2 \mu \nu}=-E_{\mu \nu}-2 b\left(Q_{\mu \nu}-\frac{1}{4} Q g_{\mu \nu}\right)+b S C_{\mu \nu},
\end{aligned}
$$

where $D, E, P$ and $Q$ are defined by

$$
\begin{aligned}
D_{\mu \nu} & \equiv e^{-1} \epsilon_{(\mu \mid}^{\alpha \beta} \nabla_{\alpha} C_{\beta \mid \nu)}, & & P_{\mu \nu} \equiv G_{\mu}{ }^{\rho} S_{\nu \rho}, \\
E_{\mu \nu} & \equiv e^{-1} \epsilon_{(\mu \mid}{ }^{\alpha \beta} \nabla_{\alpha} f_{2 \beta \mid \nu)}, & & Q_{\mu \nu} \equiv C_{(\mu}{ }^{\rho} S_{\nu) \rho} .
\end{aligned}
$$

Substituting these expressions back into the action leads to the following 'extended' NMG (ENMG) Lagrangian density

$$
\mathcal{L}_{\mathrm{ENMG}} \equiv \mathcal{L}_{4}=\frac{1}{2}\left\{\sigma R-2 \Lambda_{0}+\frac{1}{m^{2}} P+\frac{1}{m^{4}}\left(2 a \operatorname{det}(S)-b C^{\mu \nu} C_{\mu \nu}\right)\right\} .
$$

At order $1 / m^{2}$ we have the NMG combination of $R^{2}$ terms,

$$
P=R_{\mu \nu} R^{\mu \nu}-\frac{3}{8} R^{2}
$$

while at order $1 / \mathrm{m}^{4}$ we find the following two combinations of six-derivative terms:

$$
\begin{aligned}
-6 \operatorname{det}(S) & =2 R_{\mu}^{\nu} R_{\nu}^{\rho} R_{\rho}^{\mu}-\frac{9}{4} R R_{\mu \nu} R^{\mu \nu}+\frac{17}{32} R^{3}, \\
C^{\mu \nu} C_{\mu \nu} & =R_{\mu \nu} \square R^{\mu \nu}-\frac{3}{8} R \square R-3 R_{\mu}^{\nu} R_{\nu}^{\rho} R_{\rho}^{\mu}+\frac{5}{2} R R_{\mu \nu} R^{\mu \nu}-\frac{1}{2} R^{3} .
\end{aligned}
$$

The last identity is up to total derivatives. This theory is free of scalar ghosts and has four local degrees of freedom by construction, as was verified in section 2.2. 
We can systematically continue this program and find more and more higher order terms. As an example we present the eight-derivative theory:

$$
\begin{aligned}
\mathcal{L}_{6}=\mathcal{L}_{\mathrm{ENMG}}+\frac{1}{m^{6}}\{ & \kappa_{1}\left(P_{\mu \nu} P^{\mu \nu}-\frac{3}{8} P^{2}\right)+\kappa_{2}\left(S_{\mu}^{\rho} C_{\rho \nu} C^{\mu \nu}-\frac{1}{2} S C_{\mu \nu} C^{\mu \nu}\right) \\
& \left.+\kappa_{3}\left(C_{\mu \nu} \square C^{\mu \nu}+3 S_{\mu}^{\rho} C_{\rho \nu} C^{\mu \nu}+S C_{\mu \nu} C^{\mu \nu}\right)\right\},
\end{aligned}
$$

with parameters $\kappa_{1}, \kappa_{2}$ and $\kappa_{3}$. One can simplify the $\kappa_{1}$-term using the Schouten identity $\$_{\mu \nu}^{4}=\frac{1}{2}\left(\$_{\mu \nu}^{2}\right)^{2}$, where $\$$ is the traceless Schouten tensor, $\$_{\mu \nu}=S_{\mu \nu}-\frac{1}{3} S g_{\mu \nu}$, as follows:

$$
-\frac{\kappa_{1}}{12}\left(16 S S_{\mu}^{\rho} S_{\rho}^{\sigma} S_{\sigma}^{\mu}-3\left(S_{\mu \nu} S^{\mu \nu}\right)^{2}-18 S_{\mu \nu} S^{\mu \nu} S^{2}+5 S^{4}\right) .
$$

The $a$ - and $\kappa_{1}$-terms above are precisely the combination of $R^{3}$ and $R^{4}$ terms found by Sinha in [25] by demanding the presence of a holographic $c$-theorem in higher-derivative extensions of new massive gravity, see also [33]. The $b_{-}, \kappa_{2}$ - and $\kappa_{3}$-terms were not considered in their considerations regarding the holographic $c$-theorem. We will comment on this in section 4.4 .

\subsection{Linearization}

In this section we study the extended NMG theory by linearizing the model around a maximally symmetric vacuum parametrized by a background dreibein $\bar{e}$, spin-connection $\bar{\omega}$ and cosmological constant $\Lambda$ that satisfy

$$
\bar{R}^{a} \equiv \overline{\mathcal{D}} \bar{\omega}^{a}=\frac{\Lambda}{2} \epsilon_{b c}^{a} \bar{e}^{b} \bar{e}^{c}, \quad \overline{\mathcal{D}} \bar{e}^{a}=0 .
$$

All barred quantities refer to the background. The background values for the auxiliary fields can be determined by their background equations of motion. Since the parity-even and parity-odd models have the same field equations, these fields have the same background values in both models. The $f$ fields all have background values proportional to the background dreibein. The $h$ fields, which are constructed from the Cotton tensor, vanish on this background. We parametrize the vector-valued fluctuations of the one-forms around the vacuum as

$$
\begin{aligned}
e & =\bar{e}+\kappa k_{0}, & \omega & =\bar{\omega}+\kappa v_{0}, \\
f_{1} & =-\frac{\Lambda}{2} \bar{e}+\kappa k_{1}, & h_{1} & =\kappa v_{1}, \\
f_{2} & =-\frac{\Lambda}{2}\left(m^{2}+\frac{a \Lambda}{4}\right) \bar{e}+\kappa k_{2}, & h_{2} & =\kappa v_{2},
\end{aligned}
$$

where we used $\kappa$ as a small expansion parameter. We next substitute eq. (3.14) into the recursive action (2.7) and keep the quadratic terms which are $\kappa$-independent.

We first focus on the quadratic Lagrangians $L_{0}^{(2)}$ and $L_{1}^{(2)}$ corresponding to the Einstein gravity action (1.5) and the conformal gravity action (1.6) which have no propagating 
degrees of freedom. Plugging the expressions (3.14) into the actions (1.5) and (1.6) we find

$$
\begin{aligned}
& L_{0}^{(2)}=-\left\{k_{a} \overline{\mathcal{D}} v^{a}+\frac{1}{2} \epsilon_{a b c} \bar{e}^{a}\left(v^{b} v^{c}-\Lambda k^{b} k^{c}\right)\right\}, \quad \Lambda=\Lambda_{0}, \\
& L_{1}^{(2)}=\mu^{-1}\left\{k_{1 a}\left(\overline{\mathcal{D}} k^{a}+\epsilon_{a b c} \bar{e}^{b} v^{c}\right)+\frac{1}{2} v_{a}\left(\overline{\mathcal{D}} v^{a}-\Lambda \epsilon_{a b c} \bar{e}^{b} k^{c}\right)\right\},
\end{aligned}
$$

where we have defined $k \equiv k_{0}$ and $v \equiv v_{0}$. By using the field equations for $v^{a}$ and $k_{1}^{a}$ it is possible to eliminate them in $L_{0}^{(2)}$ and $L_{1}^{(2)}$. We thus obtain the quadratic Lagrangian density in the following more conventional second order form:

$$
\mathcal{L}_{0}^{(2)}=-k^{\mu \nu} \mathcal{G}_{\mu \nu}(k) \quad \text { and } \quad \mathcal{L}_{1}^{(2)}=-\frac{1}{\mu} \epsilon^{\mu \alpha}{ }_{\rho} \bar{\nabla}_{\alpha} k^{\rho \nu} \mathcal{G}_{\mu \nu}(k) .
$$

The corresponding linear field equations are given by

$$
\mathcal{G}_{\mu \nu}(k)=0 \quad \text { and } \quad\left(\mathcal{D}^{0} \mathcal{G}(k)\right)_{\mu \nu}=0 .
$$

Here $\mathcal{G}_{\mu \nu}(k)$ is the linearized Einstein tensor invariant under linear diffeomorphisms. In the transverse traceless gauge we have,

$$
\mathcal{G}_{\mu \nu}(k)=-\frac{1}{2}(\bar{\square}-2 \Lambda) k_{\mu \nu} .
$$

Clearly, $\mathcal{L}_{0}^{(2)}$ is the linearized Einstein-Hilbert term without any propagating degrees of freedom. On the other hand, in $\mathcal{L}_{1}^{(2)}$ there is an additional partially massless mode satisfying

$$
\left(\mathcal{D}^{0} k\right)_{\mu \nu}=\epsilon_{\mu}{ }^{\alpha \rho} \bar{\nabla}_{\alpha} k_{\rho \nu}=0 .
$$

This equation is invariant under an additional linearized Weyl transformation [15]. Below, we discuss the linearized theories of the parity-even sector up to $S_{4}$ and confirm that the addition of two auxiliary fields adds a massive spin-2 mode. For a similar analysis of the parity-odd sector we refer to appendix B.

Moving to NMG, we consider the four derivative action $S_{2}$, see eq. (1.7), where we have included the auxiliary form fields $f_{1}$ and $h_{1}$. The background field equation enforces the following quadratic relation for $\Lambda$ :

$$
\Lambda_{0}=\Lambda\left(\sigma+\frac{\Lambda}{4 m^{2}}\right)
$$

Making the field redefinition, $k_{1} \rightarrow k_{1}-\frac{\Lambda}{2} k$, the quadratic Lagrangian three-form reads,

$$
\begin{aligned}
L_{2}^{(2)}=\left(\sigma-\frac{\Lambda}{2 m^{2}}\right) L_{0}^{(2)}-\frac{1}{m^{2}}\{ & k_{1 a}\left(\overline{\mathcal{D}} v^{a}-\Lambda \epsilon^{a}{ }_{b c} \bar{e}^{b} k^{c}+\frac{1}{2} \epsilon^{a} b c \bar{e} k_{1}^{c}\right) \\
& \left.+v_{1 a}\left(\overline{\mathcal{D}} k^{a}+\epsilon^{a}{ }_{b c} \bar{e}^{b} v^{c}\right)\right\},
\end{aligned}
$$

where $L_{0}^{(2)}$ is given in eq. (3.15). Eliminating $v^{a}$ and $v_{1}^{a}$ using their equations of motion, the Lagrangian reduces to the following density:

$$
\begin{aligned}
\mathcal{L}_{2}^{(2)} & =-\sigma_{2} k^{\mu \nu} \mathcal{G}_{\mu \nu}(k)-\frac{2}{m^{2}} k_{1}^{\mu \nu} \mathcal{G}_{\mu \nu}(k)-\frac{1}{2 m^{2}}\left(k_{1 \mu \nu} k_{1}{ }^{\mu \nu}-k_{1}^{2}\right), \\
& =\sigma_{2} \mathcal{L}_{0}^{(2)}-\frac{1}{m^{4} \sigma_{2}} \mathcal{L}_{\mathrm{FP}}\left(k_{1}, \mathcal{M}\right), \quad \sigma_{2}=\sigma-\frac{\Lambda}{2 m^{2}}=-\frac{\mathcal{M}^{2}}{m^{2}}
\end{aligned}
$$


The Fierz-Pauli Lagrangian $\mathcal{L}_{\mathrm{FP}}$ is given by

$$
\mathcal{L}_{\mathrm{FP}}(\tilde{k}, \mathcal{M})=-\tilde{k}^{\mu \nu} \mathcal{G}_{\mu \nu}(\tilde{k})-\frac{1}{2} \mathcal{M}^{2}\left(\tilde{k}^{\mu \nu} \tilde{k}_{\mu \nu}-\tilde{k}^{2}\right)
$$

for a generic massive mode $\tilde{k}$. Assuming that $\sigma_{2} \neq 0$, the Lagrangian density $\mathcal{L}_{2}^{(2)}$ has been diagonalized by a proper shift in the $k$ field;

$$
k_{\mu \nu} \rightarrow k_{\mu \nu}+\frac{1}{m^{2} \sigma_{2}} k_{1 \mu \nu}
$$

To avoid tachyons the mass of the massive mode should be bounded as $\mathcal{M}^{2} \geq 0$ (see for instance [35, 36]). In order to avoid ghosts both kinetic terms in (3.23) should come with the same sign. This already shows that depending on the sign of $\sigma_{2}$, either the massless mode or the massive mode in (3.23) is ghost-like. The massless mode should not be ghostlike as it determines the charges for the gravitational theory through the Brown-York stress tensor. We therefore take $\sigma_{2}>0$. However, restricting to $\sigma_{2}>0$ results in a massive spin-2 ghost. As we will see later this is a general problem in higher-derivative theories, denoted as the bulk-boundary unitarity clash.

We now go one step further and consider the quadratic part of the action $S_{4}$. The cosmological parameter $\Lambda_{0}$ is now related to the physical cosmological constant $\Lambda$ by the cubic equation

$$
\Lambda_{0}=\Lambda\left(\sigma+\frac{\Lambda}{4 m^{2}}+\frac{a \Lambda^{2}}{8 m^{4}}\right)
$$

By performing the field redefinition

$$
k_{2} \rightarrow k_{2}-\frac{\Lambda}{2}\left(m^{2}+\frac{a \Lambda}{4}\right) k, \quad k_{1} \rightarrow k_{1}-\frac{\Lambda}{2} k, \quad v_{2} \rightarrow v_{2}+\frac{b \Lambda}{2} v_{1},
$$

the quadratic Lagrangian three-form part of $S_{4}$ can be brought into the following form:

$$
\begin{aligned}
L_{4}^{(2)}= & \left(\sigma-\frac{\Lambda}{2 m^{2}}-\frac{a \Lambda^{2}}{8 m^{4}}\right) L_{0}^{(2)}+\frac{1}{2 m^{2}}\left(1+\frac{a \Lambda}{2 m^{2}}\right) \epsilon_{a b c} \bar{e}^{a} k_{1}{ }^{b} k_{1}^{c} \\
-\frac{1}{m^{4}}\{ & k_{2 a}\left(\overline{\mathcal{D}} v^{a}-\Lambda \epsilon^{a}{ }_{b c} \bar{e}^{b} k^{c}+\epsilon^{a}{ }_{b c} \bar{e}^{b} k_{1}^{c}\right)+v_{2 a}\left(\overline{\mathcal{D}} k^{a}+\epsilon^{a}{ }_{b c} \bar{e}^{b} v^{c}\right) \\
& \left.\quad-b v_{1 a}\left(\overline{\mathcal{D}} k_{1}^{a}+\frac{1}{2} \epsilon_{b c}^{a} \bar{e}^{b} v_{1}^{c}\right)\right\} .
\end{aligned}
$$

Upon eliminating the auxiliary fields $v^{a}, v_{1}{ }^{a}$ and $v_{2}{ }^{a}$ by using their equations of motion, the quadratic Lagrangian density may be written as

$$
\begin{aligned}
\mathcal{L}_{4}^{(2)}= & -\sigma_{4} k^{\mu \nu} \mathcal{G}_{\mu \nu}(k)-\frac{2}{m^{4}} k_{2}^{\mu \nu} \mathcal{G}_{\mu \nu}(k)-\frac{b}{m^{4}} k_{1}^{\mu \nu} \mathcal{G}_{\mu \nu}\left(k_{1}\right) \\
& +\frac{1}{2 m^{2}} \Theta\left(k_{1}^{\mu \nu} k_{1 \mu \nu}-k_{1}^{2}\right)-\frac{1}{m^{4}}\left(k_{1}^{\mu \nu} k_{2 \mu \nu}-k_{1} k_{2}\right),
\end{aligned}
$$

where

$$
\sigma_{4}=\sigma-\frac{\Lambda}{2 m^{2}}-\frac{a \Lambda^{2}}{8 m^{4}} \quad \text { and } \quad \Theta=1+\frac{a \Lambda}{2 m^{2}}-\frac{b \Lambda}{m^{2}}
$$


For general values of the parameters this quadratic Lagrangian leads to a sixth order differential equation for $k_{\mu \nu}$. The matrix for the kinetic terms and the mass terms in the basis defined by $|k\rangle, m^{2}\left|k_{1}\right\rangle$ and $m^{4}\left|k_{2}\right\rangle$ can be written as,

$$
\mathcal{K}=-\left(\begin{array}{ccc}
\sigma_{4} & 0 & 1 \\
0 & b & 0 \\
1 & 0 & 0
\end{array}\right) \quad \text { and } \quad \mathcal{M}^{2}=m^{2}\left(\begin{array}{ccc}
0 & 0 & 0 \\
0 & -\Theta & 1 \\
0 & 1 & 0
\end{array}\right)
$$

Assuming that $\sigma_{4} \neq 0$ and $b \neq 0$ these two matrices can be diagonalized simultaneously by redefining the fields

$$
\begin{aligned}
k & =k^{0}-\frac{1}{\sigma_{4}}\left(k^{+}-k^{-}\right), \\
k_{1} & =-\frac{m^{4}}{b}\left(\frac{1}{\mathcal{M}_{-}^{2}} k^{+}-\frac{1}{\mathcal{M}_{+}^{2}} k^{-}\right), \\
k_{2} & =m^{4}\left(k^{+}-k^{-}\right),
\end{aligned}
$$

such that the quadratic Lagrangian becomes the sum of the linearized Einstein-Hilbert term and two Fierz-Pauli terms

$$
\mathcal{L}_{4}^{(2)}=\sigma_{4} \mathcal{L}_{0}^{(2)}\left(k^{0}\right)+\mathcal{K}_{+} \mathcal{L}_{\mathrm{FP}}\left(k^{+}, \mathcal{M}_{+}\right)+\mathcal{K}_{-} \mathcal{L}_{\mathrm{FP}}\left(k^{-}, \mathcal{M}_{-}\right) .
$$

Here $\sigma_{4}$ and $\mathcal{K}_{ \pm}$satisfy

$$
\sigma_{4} \mathcal{K}_{+} \mathcal{K}_{-}=-\frac{\Theta^{2}-4 b \sigma_{4}}{b \sigma_{4}^{2}}=\mathcal{K}_{+}+\mathcal{K}_{-},
$$

while the Fierz-Pauli masses $\mathcal{M}_{ \pm}^{2}$ are given by

$$
\mathcal{M}_{+}^{2} \mathcal{M}_{-}^{2}=\frac{m^{4}}{b} \sigma_{4} \quad \text { and } \quad \mathcal{M}_{+}^{2}-\mathcal{M}_{-}^{2}=\frac{m^{2}}{b} \sqrt{\Theta^{2}-4 b \sigma_{4}} .
$$

The numerator in (3.36) should be positive, otherwise the masses in (3.37) become imaginary. Like in the previous case, we see that it is not possible to achieve a positive sign for the kinetic terms and the masses simultaneously. From (3.36) and (3.37) we see that there is always either a negative mass squared (when $b \sigma_{4}<0$ ) or a wrong-sign kinetic term (when $b \sigma_{4}>0$ ) in the theory. For all values where $\sigma_{4} \neq 0$ or $b \neq 0$, one of the massive modes is either tachyonic or a ghost. In the next section we will discuss what happens at special points in the parameter space.

\subsection{Critical lines and the tricritical point}

In the above analysis we disregarded the points in the parameter space that reduce the rank of the two matrices given in eq. (3.31). Below we discuss these special points separately.

$\boldsymbol{b}=\mathbf{0}$ : At this point the rank of the matrix $\mathcal{K}$ is reduced by one. The action (3.1) is now independent of the auxiliary field $h_{1}$, and $f_{2}$ is algebraically given in terms of $f_{1}$. This reduces the number of local degrees of freedom from four to two, representing a single massive graviton. From eq. (3.8) we see that the term involving the Cotton tensor has disappeared and the action reduces to the 'cubic extended' NMG model described in [25]. 
$\boldsymbol{b}=\boldsymbol{\Theta}=\mathbf{0}$ : At this special point the linearized equations become second order in derivatives and the massive mode disappears from the linearized spectrum. Note that there are no ghosts left in the linearized theory [25]. It is however not clear if this feature survives at the non-linear level.

$\sigma_{4}=0$ : At this critical line one of the FP masses vanishes, but the linearized equations remain sixth-order in derivatives. Consequently a new, logarithmic (log)-mode appears and together with the massless mode it forms a Jordan cell of rank two. The Lagrangian (3.29) is not diagonalizable any more.

$\sigma_{4}=\Theta=0$ : This is a 'tricritical' point, where both FP masses vanish and the corresponding massless gravitons form a Jordan cell of rank three. The spectrum now contains one log-mode and a $\log ^{2}$-mode (see for instance $[26,40]$ ).

$\Theta^{2}=4 \boldsymbol{b} \sigma_{4}$ : This is another critical line where the two non-zero FP masses degenerate and form a Jordan cell of rank two. At this point the spectrum contains one massive mode and a massive log-mode.

Among the above critical points, $\sigma_{4}=0$ and $\Theta=0$ can only occur when $\Lambda \neq 0$. They are interesting from the AdS/CFT point of view. Especially the $b=\Theta=0$ point is interesting as the linearized analysis suggests perturbative unitarity. For a more detailed treatment of the $\sigma_{4}=0$ case, see section 4.3 and also the reference [26] where most of the computations performed in the context of the AdS/LCFT correspondence carry over to this model.

\section{Anti-de Sitter holography}

All the extended massive gravity models we constructed so far admit an AdS vacuum. Hence, it is possible to study their holographic dual by imposing suitable asymptotically AdS boundary conditions. The asymptotic symmetry algebra emerges as the algebra of conserved global charges related to gauge transformations which preserve the AdS boundary conditions. The procedure for finding the asymptotic symmetry algebra is similar to that of pure CS gauge theories on manifolds with a boundary [37]. The main difference is that for CS-like theories not all constraint functions generate gauge symmetries. The first step is to identify the first class constraints and their corresponding boundary terms for the CS-like theories we have discussed in the preceding section. This is done in full detail in appendix A. Here we will briefly summarize the main results derived there and continue with a discussion on the suitable boundary conditions and the asymptotic symmetry transformations which preserve them. After deriving the central charge in the asymptotic symmetry algebra of extended NMG, we discuss the appearance of Jordan cells at special points in its parameter space where the central charge vanishes and compute the new logarithmic anomalies. We conclude this section by showing how the models constructed in this paper are consistent with a holographic $c$-theorem. 


\subsection{Gauge symmetries in CS-like theories}

In appendix A we have identified, using standard techniques, the first class constraints $\phi_{\text {LL }}$ and $\phi_{\text {diff }}$ of a general CS-like model that generate local Lorentz transformations and diffeomorphisms, see eqs. (A.13) and (A.15), respectively. On the AdS background, these can be written in terms of a set of mutually commuting $\mathrm{SL}(2, \mathbb{R})$ generators $J_{ \pm}$with Poisson bracket algebra

$$
\left\{J_{ \pm}[\xi], J_{ \pm}[\eta]\right\}=J_{ \pm}[\xi \times \eta]+\text { B.T. }, \quad\left\{J_{+}[\xi], J_{-}[\eta]\right\}=0
$$

In general, the presence of a boundary introduces non-trivial boundary terms in the definition of the $J_{ \pm}$and in the Poisson bracket algebra (4.1). In appendix A we show that for the CS-like theories we consider here, the improved generators $\mathcal{J}_{ \pm}$defined by

$$
\mathcal{J}_{ \pm}\left[\xi^{ \pm}\right]=J_{ \pm}\left[\xi^{ \pm}\right]+Q_{ \pm}\left[\xi^{ \pm}\right]
$$

are differentiable provided that the variation of the boundary term $Q_{ \pm}$takes the form

$$
\delta Q_{ \pm}\left[\xi^{ \pm}\right]=\frac{\hat{k}}{2 \pi} \int_{\partial \Sigma} d x^{i} \xi_{a}^{ \pm}\left(\delta \omega_{i}^{a} \pm \frac{1}{\ell} \delta e_{i}^{a}\right)
$$

Here $\hat{k}$ is an effective coupling which depends on the specific theory under consideration. The Poisson brackets of the improved generators then pick up a boundary term which can be derived from the general formula (A.9) in appendix A:

$$
\left\{\mathcal{J}_{ \pm}\left[\xi^{ \pm}\right], \mathcal{J}_{ \pm}\left[\eta^{ \pm}\right]\right\}=\cdots+\frac{\hat{k}}{4 \pi} \int_{\partial \Sigma} d x^{i} \xi^{ \pm a}\left[\partial_{i} \eta_{a}^{ \pm}+\epsilon_{a b c}\left(\omega_{i}^{b} \pm \frac{1}{\ell} e_{i}^{b}\right) \eta^{ \pm c}\right]
$$

Here the dots denote bulk terms. After adopting suitable boundary conditions, the charges become integrable and the above boundary term will provide a term needed to improve the bulk part in (4.1) and a central extension. We can now discuss the AdS boundary conditions.

\subsection{AdS boundary conditions and the central charge}

To give the boundary conditions, it is convenient to represent the spin-connection and the dreibein of the AdS background with radius $\ell$ in the following combinations:

$$
\begin{aligned}
& \bar{\omega}+\frac{\bar{e}}{\ell}=b^{-1}\left(L_{1}+\frac{1}{4} L_{-1}\right) b d x^{+}+b^{-1} \partial_{\rho} b d \rho, \\
& \bar{\omega}-\frac{\bar{e}}{\ell}=-b\left(L_{-1}+\frac{1}{4} L_{1}\right) b^{-1} d x^{-}+b \partial_{\rho} b^{-1} d \rho,
\end{aligned}
$$

where $x^{ \pm}=\frac{t}{\ell} \pm \varphi, b=e^{\rho L_{0}}$ and,

$$
L_{1}=J_{0}+J_{1}, \quad L_{-1}=J_{0}-J_{1} \quad \text { and } \quad L_{0}=J_{2} .
$$

where the $J_{a}$ are the $\mathrm{SO}(2,1)$ generators defined in (1.3). The AdS boundary conditions are presented in terms of some free state dependent normalizable contributions to this 
background. These contributions behave as the vacuum expectation value (VEV) for the boundary operators which are sourced by non-normalizable modes. As we discussed before, each pair $\left(f_{I}, h_{I}\right)$ introduces two new degrees of freedom representing a massive spin-2 normalizable mode with mass $\mathcal{M}^{2}$ which satisfy the following equation,

$$
\tilde{\mathcal{D}}^{M} \mathcal{D}^{M} k_{\mu \nu}^{I}=0
$$

with

$$
\left(\mathcal{D}^{M}\right)_{\mu}^{\nu}=\delta_{\mu}^{\nu}+\frac{1}{M} \epsilon_{\mu}^{\tau \nu} \bar{\nabla}_{\tau}, \quad\left(\tilde{\mathcal{D}}^{M}\right)_{\mu}^{\nu}=\delta_{\mu}^{\nu}-\frac{1}{M} \epsilon_{\mu}^{\tau \nu} \bar{\nabla}_{\tau}
$$

and $M^{2} \ell^{2}=\mathcal{M}^{2} \ell^{2}+1$. The non-normalizable partner of this mode plays the role of a source which couples to a new operator $\mathcal{O}_{I}$ in the dual conformal field theory with conformal weights $(h, \tilde{h})$. These weights are related to the mass and angular momentum of the bulk mode via the relations [38]

$$
\Delta=h+\tilde{h}=1+|M \ell| \quad \text { and } \quad s=h-\tilde{h}= \pm 2,
$$

where \pm distinguishes between the right and left sectors. The requirement of unitarity bounds the scaling dimension $\Delta$ as $\Delta \geq|s|$ (when $1 \leq \Delta<|s|$ one of the conformal weights $(h, \tilde{h})$ is negative). When the masses of $N$ modes degenerate, the conformal weights of their corresponding operators also degenerate and they form a Jordan cell of rank- $N$ in a logarithmic conformal field theory.

In this work we are interested in the asymptotic symmetry algebra generated by the gauge symmetries of the bulk theory. For this reason, we will not consider the sources for the massive modes. The resulting AdS boundary conditions are called Brown-Henneaux boundary conditions. These boundary conditions are sufficient for finding the Poisson brackets between the gauge generators [19]. In the first order formalism, inspired by the Chern-Simons formulation of 3D gravity, the Brown-Henneaux boundary conditions on the dreibein and the spin-connection are given by [39]

$$
\begin{aligned}
& \omega+\frac{e}{\ell}=b^{-1}\left\{\left(L_{1}+\mathcal{L}\left(x^{+}\right) L_{-1}\right) d x^{+}+\mathrm{d}\right\} b, \\
& \omega-\frac{e}{\ell}=-b\left\{\left(L_{-1}+\tilde{\mathcal{L}}\left(x^{-}\right) L_{1}\right) d x^{-}-\mathrm{d}\right\} b^{-1} .
\end{aligned}
$$

The state dependent functions $\mathcal{L}\left(x^{+}\right)$and $\tilde{\mathcal{L}}\left(x^{-}\right)$are the vacuum expectation value (VEV) of the boundary energy-momentum operator.

In order to integrate the expression (4.3) to the boundary charges, we impose the Brown-Henneaux boundary conditions (4.10) on the dreibein and the spin-connection. The gauge transformations preserving these boundary conditions are then given by:

$$
\xi^{+}=b^{-1} \epsilon\left(x^{+}\right) b \quad \text { and } \quad \xi^{-}=b \tilde{\epsilon}\left(x^{-}\right) b^{-1},
$$

where $^{7}$

$$
\epsilon^{-1}=\frac{1}{2} \epsilon^{\prime \prime}+\epsilon \mathcal{L}, \quad \epsilon^{0}=-\epsilon^{\prime} \quad \text { with } \quad \epsilon^{1} \equiv \epsilon .
$$

\footnotetext{
${ }^{7}$ Since we are dealing with parity preserving models, we concentrate only on the left sector. The right sector will be determined via a parity transformation.
} 
The variation of the state-dependent function $\mathcal{L}$ in eq. (4.10) with respect to $\epsilon$, the parameter of the symmetry transformation, is given by,

$$
\delta_{\epsilon} \mathcal{L}\left(x^{+}\right)=\epsilon\left(x^{+}\right) \mathcal{L}^{\prime}\left(x^{+}\right)+2 \epsilon^{\prime}\left(x^{+}\right) \mathcal{L}\left(x^{+}\right)+\frac{1}{2} \epsilon^{\prime \prime \prime}\left(x^{+}\right)
$$

This leads to the following expression for the conserved charge $Q=Q_{+}+Q_{-}$at the boundary:

$$
Q=\frac{\hat{k}}{2 \pi} \int d \varphi\left[\epsilon\left(x^{+}\right) \mathcal{L}\left(x^{+}\right)-\tilde{\epsilon}\left(x^{-}\right) \tilde{\mathcal{L}}\left(x^{-}\right)\right] .
$$

We can now compute the Poisson brackets (4.1) with the boundary term (4.4) after suitably identifying $\xi$ and $\eta$ using eq. (4.11) and defining the generators as,

$$
L_{n}=\mathcal{J}_{+}\left[\epsilon=e^{i n x^{+}}\right] \quad \text { and } \quad \tilde{L}_{n}=\mathcal{J}_{-}\left[\tilde{\epsilon}=e^{i n x^{-}}\right] .
$$

As expected we find two copies of Virasoro algebra,

$$
\begin{aligned}
& i\left\{L_{m}, L_{n}\right\}=(m-n) L_{m+n}+\frac{c}{12} m\left(m^{2}-1\right) \delta_{m+n, 0}, \\
& i\left\{\tilde{L}_{m}, \tilde{L}_{n}\right\}=(m-n) \tilde{L}_{m+n}+\frac{\tilde{c}}{12} m\left(m^{2}-1\right) \delta_{m+n, 0},
\end{aligned}
$$

where $c=\tilde{c}=6 \hat{k}$ for parity-even models and $c=-\tilde{c}=6 \hat{k}$ for the parity-odd theories. In appendix A we explicitly compute $\hat{k}$ for the cubic extended NMG model defined by eq. (3.1), leading to the central charge

$$
c^{\mathrm{ENMG}}=\frac{3 \ell}{2 G} \sigma_{4}=\frac{3 \ell}{2 G}\left(\sigma+\frac{1}{2 m^{2} \ell^{2}}-\frac{a}{8 m^{4} \ell^{4}}\right) .
$$

The central charge is proportional to the earlier defined parameter $\sigma_{4}$. Even though the parameter $b$ does not appear in the expression for the central charge, it does play a role in the analysis of the critical lines and points.

Appendix B deals with the parity-odd extension of $S_{1}$ which is denoted as $S_{3}$ in (2.9). Using the results derived there, we find

$$
c_{3}=-\tilde{c}_{3}=\frac{3}{2 \mu G} .
$$

This expression is equivalent to the central charge in conformal gravity; the higherderivative terms in (2.9) do not contribute to the central charge. We will address this point in more detail in section 4.4 .

\subsection{Logarithmic anomalies}

The linearized analysis in section 3.1 showed that the presence of massive spin-2 ghosts or tachyons in a general extended new massive gravity model cannot be avoided for non-zero FP masses. However, there are critical points where one or both of the FP masses vanish. At these points where the central charge also vanishes, new logarithmic modes appear and the linear theory is no longer diagonalisable. In that case the gravitational theory is conjectured to be dual to a logarithmic conformal field theory (LCFT). Knowledge of the 


\begin{tabular}{|c|c|}
\hline & $(h, \tilde{h})$ \\
\hline$T(z)$ & $(2,0)$ \\
\hline $\mathcal{O}_{ \pm}(z, \bar{z})$ & $\left(\frac{3}{2}+\frac{1}{2} \sqrt{1+\ell^{2} \mathcal{M}_{ \pm}^{2}},-\frac{1}{2}+\frac{1}{2} \sqrt{1+\ell^{2} \mathcal{M}_{ \pm}^{2}}\right)$ \\
\hline
\end{tabular}

Table 1. This table indicates the conformal weights of the operators $T(z)$ and $\mathcal{O}_{ \pm}(z, \bar{z})$. The expressions for $\mathcal{M}_{ \pm}$can be obtained from eq. (3.37).

central charge and the weights of the bulk modes is sufficient to fix the structure of the two-point functions at the critical line and at the tricritical point [40]. Here we concentrate on the left-moving sector whose spectrum is given in table 1.

Similar results hold for the right-moving sector as the two sectors are related by a parity transformation $h \leftrightarrow \tilde{h}$. Criticality happens whenever the conformal weights of these operators degenerate with the conformal dimension of the energy momentum tensor $\left(h_{ \pm}=2\right) .{ }^{8}$ In the non-critical case, the two-point function of the left-moving components of the boundary stress tensor $T(z)$ is given by

$$
\langle T(z) T(0)\rangle=\frac{c}{2 z^{4}},
$$

where $c$ is given by eq. (4.18). If we tune $a$ to its critical value $a_{\text {crit }}$, defined such that $\sigma_{4}=0$ and the central charge (4.18) vanishes;

$$
a=a_{\text {crit }}=4 \ell^{2} m^{2}\left(1+2 \ell^{2} m^{2} \sigma\right),
$$

then one of the two masses vanishes, which we take to be $\mathcal{M}_{-}$, and its corresponding boundary operator becomes the logarithmic partner of $T$. They form a Jordan cell of rank 2 with the following two-point functions,

$$
\begin{aligned}
& \langle T(z) T(0)\rangle=0 \\
& \left\langle T(z) \mathcal{O}_{1}(0)\right\rangle=\frac{B_{1}}{2 z^{4}} \\
& \left\langle\mathcal{O}_{1}(z, \bar{z}) \mathcal{O}_{1}(0)\right\rangle=-\frac{B_{1} \log |z|^{2}}{z^{4}} .
\end{aligned}
$$

The new anomaly $B_{1}$ can be computed through the limiting procedure of [40];

$$
B_{1}=\lim _{a \rightarrow a_{\text {crit }}} \frac{c}{2-h_{-}}=-\frac{24 \ell}{G}\left(\sigma+\frac{1}{4 m^{2} \ell^{2}}-\frac{b}{4 m^{4} \ell^{4}}\right) .
$$

Note that in the limit $b \rightarrow 0$ and $a_{\text {crit }} \rightarrow 0$ the cubic extended NMG model reduces to the critical NMG model and the result (4.23) agrees with the new anomaly of NMG found in $[41,42]$.

The last case we consider is the one where $b$ also takes a critical value $b=b_{\text {crit }}=$ $\ell^{2} m^{2}\left(1+4 \ell^{2} m^{2} \sigma\right)$ such that $B_{1}=0$. This defines the tricritical point where $\sigma_{4}=\Theta=0$

\footnotetext{
${ }^{8}$ In principal one would also expect a logarithmic behavior when the two masses degenerate and hence $h_{+}=h_{-} \neq 2$. This might define a LCFT with a non-zero central charge.
} 
and both FP masses vanish. At this point, we conjecture that the correlators are those of a rank-3 LCFT with zero central charges:

$$
\begin{aligned}
& \langle T(z) T(0)\rangle=\left\langle T(z) \mathcal{O}_{1}(0)\right\rangle=0, \\
& \left\langle T(z) \mathcal{O}_{2}(0)\right\rangle=\left\langle\mathcal{O}_{1}(z) \mathcal{O}_{1}(0)\right\rangle=\frac{B_{2}}{2 z^{4}}, \\
& \left\langle\mathcal{O}_{1}(z, \bar{z}) \mathcal{O}_{2}(0)\right\rangle=-\frac{B_{2} \log |z|^{2}}{z^{4}}, \\
& \left\langle\mathcal{O}_{2}(z, \bar{z}) \mathcal{O}_{2}(0)\right\rangle=\frac{B_{2} \log ^{2}|z|^{2}}{z^{4}} .
\end{aligned}
$$

Here $\mathcal{O}_{1}(z, \bar{z})$ and $\mathcal{O}_{2}(z, \bar{z})$ are the two logarithmic partners of $T(z)$. The new anomaly $B_{2}$ at the tricritical point is obtained via a second limit:

$$
B_{2}=\lim _{b \rightarrow b_{\text {crit }}} \frac{B_{1}}{2-h_{+}}=\frac{96 \ell}{G}\left(\sigma+\frac{1}{4 m^{2} \ell^{2}}\right) .
$$

Note that after fixing $a$ and $b$ to their critical values, the free parameter $m^{2}$ is undetermined in the expression for $B_{2}$. This implies that tricritical cubic extended NMG in fact has a continuous line of tricritical points dual to a family of rank-3 LCFT's with different values for the new anomaly (4.25) in contrast with the PET gravity model of [26].

\subsection{Holographic $c$-theorem}

It is well-known that the RG flows between fixed points in a matter theory with stress tensor $\mathcal{T}_{\mu \nu}$ coupled to gravity and with AdS vacua can be described by a metric of the form

$$
d s^{2}=e^{2 \mathcal{A}(r)}\left(-d t^{2}+d \mathbf{x}^{2}\right)+d r^{2} .
$$

Assuming that the null energy condition holds for the matter sector, i.e. $\mathcal{T}_{\mu \nu} \xi^{\mu} \xi^{\nu} \geq 0$ for any null vector $\xi$, it was shown in ref. [9] that a monotonically increasing holographic $c$ function can be found in terms of $\mathcal{A}(r)$, such that it satisfies Zamolodchikov's $c$-theorem with the radial coordinate $r$ as the measure of the energy. The null energy condition now simplifies to

$$
-\mathcal{T}_{t}^{t}+\mathcal{T}_{r}^{r} \geq 0, \quad \text { for } \quad\left(\xi^{t}, \xi^{r}, \xi^{x}\right)=\left(e^{-\mathcal{A}}, 1,0\right) .
$$

A monotonically increasing holographic $c$-function can then be obtained from

$$
c^{\prime}(r)=-\frac{\mathcal{T}_{t}^{t}-\mathcal{T}_{r}^{r}}{\kappa^{2} \mathcal{A}^{\prime 2}} \geq 0 .
$$

Assuming field equations in the bulk, $\mathcal{E}_{\mu \nu}=\kappa^{2} \mathcal{T}_{\mu \nu}$, the null energy condition can equivalently be written as $\mathcal{E}_{\mu \nu} \xi^{\mu} \xi^{\nu} \geq 0$. In $[10,25]$ it is argued that one way to make $c^{\prime}(r)$ fulfill the inequality (4.28) is to have $c(r)$ be only a function of $\mathcal{A}^{\prime}$ which implies that $\mathcal{E}_{t}^{t}-\mathcal{E}_{r}^{r}$ should only be a function of $\mathcal{A}^{\prime}$ and $\mathcal{A}^{\prime \prime}$. They used this logic to constrain higher-derivative interactions by demanding the presence of such a monotonically increasing function.

Here we show that the construction (2.6) is consistent with this assumption. The ansatz (4.26) is conformal to AdS spacetime which is an Einstein metric and all solutions of the Einstein equations in three dimensions are also solutions of $C_{\mu \nu}=0$. This has the following two consequences: 
1. All fields which are constructed from the Cotton tensor and its derivatives are zero on the background (4.26). In other words, all $h$-fields and $\mathcal{D} f$ terms become zero on the ansatz (4.26), which means all equations (2.6) reduce to a set of algebraic equations among the $f$-fields in terms of the Schouten tensor which is second order in derivatives of metric. Hence the bulk field equations involve only $\mathcal{A}^{\prime}$ and $\mathcal{A}^{\prime \prime}$ by construction.

2. Consequently, we can afford terms in the action constructed from the Cotton tensor as higher-derivative corrections without affecting the $c$-function. This also suggests that the only consistent way to include $\nabla R$ terms in the action is to use the Cotton tensor.

Now if we only focus on the bulk actions (2.7) and (2.8), this suggests that terms containing $h$-fields and $\mathcal{D} f$-terms do not directly contribute to one-point functions around the AdS vacuum. The variation of the action (2.7) around the background (4.26) is only affected by $\langle\bar{f} \wedge \bar{f} \wedge \delta f\rangle$-terms because fluctuations in other terms are always proportional to a power of $\bar{h}$ - or $\overline{\mathcal{D}} \bar{f}$-term which is zero for (4.26). In the metric formulation this means that the linearized theory around (4.26) is not affected by terms where graviton fluctuations are proportional to a power of the Cotton tensor which is zero for this background. ${ }^{9}$ In fact, this is confirmed by direct calculation of the central charge for the first few parity-even models (3.8) and (3.11),

$$
c_{\mathrm{even}}=\frac{3 \ell}{2 G}\left(\sigma+\frac{1}{2 m^{2} \ell^{2}}-\frac{a}{8 m^{4} \ell^{4}}+\frac{\kappa_{1}}{16 m^{6} \ell^{6}}+\cdots\right) .
$$

The dots refer to higher-derivative contributions to the central charge. By the same reasoning, the variation of the action (2.8) around the background (4.26) is only affected by $\langle\bar{f} \wedge \bar{f} \wedge \delta h\rangle$-terms. But this term is also zero for any maximally symmetric spacetime such as AdS. In the metric formulation this is more transparent from the fact that $\bar{g}^{\mu \nu} \delta C_{\mu \nu}=0$. So the interaction terms in the odd sector do not contribute to the central charge either. This means that the central charge in the parity-odd models is universal (B.21) and not affected by any higher-derivative term,

$$
c_{\text {odd }}=-\tilde{c}_{\text {odd }}=\frac{3}{2 \mu G} .
$$

We conclude that only interaction terms constructed solely from the Schouten tensor can contribute to the central charge. This is consistent with earlier studies of the holographic $c$-theorem in this context [33]. Terms involving the Cotton tensor are allowed by the holographic $c$-theorem but do not contribute to the central charge - these terms however can contribute to the two point functions as we saw in section 4.3.

\footnotetext{
${ }^{9}$ The value of the central charge is not fully determined by the equations of motion. There is always a total derivative ambiguity which should be fixed by adding suitable boundary terms to the action and imposing suitable boundary conditions.
} 


\section{Discussion}

In this paper, we proposed a systematic procedure of constructing higher-derivative extensions of 3D general relativity which are free of scalar ghost degrees of freedom and propagate massive spin- 2 excitations. The absence of the scaler ghosts ensures that the only degrees of freedom involved in the theory are spin- 2 modes interacting with a single massless spin-two field. We have used the Chern-Simons-like formulation of 3D gravity with auxiliary form fields to find these specific scalar ghost-free combinations, which we gave explicitly up to eighth order in derivatives of the metric. These combinations turn out to be consistent with supersymmetry [43] and a holographic $c$-theorem. The number of these propagating spin-2 degrees of freedom - some of which may be ghosts - is determined by the number of auxiliary fields we introduce. We considered only theories which can be written in terms of a single metric and with a finite number of ghost-free combinations for CS-like theories without a single-metric action see [36, 44, 45].

Born-Infeld gravity. One can also construct single-metric theories with an infinite number of ghost-free terms; as an example we consider the following extension of NMG with a $\langle f \wedge f \wedge f\rangle$-term in its CS-like formulation:

$$
L=-\sigma e_{a} R^{a}+\frac{\Lambda_{0}}{6} \epsilon_{a b c} e^{a} e^{b} e^{c}+h^{a} \mathcal{D} e_{a}-\frac{1}{m^{2}} f_{a}\left(R^{a}+\frac{1}{2} \epsilon_{b c}^{a} e^{b} f^{c}+\frac{a}{6 m^{2}} \epsilon_{b c}^{a} f^{b} f^{c}\right),
$$

where $a$ is a free dimensionless parameter. The field equations obtained by varying w.r.t. $f$ are given by

$$
R^{a}+\epsilon_{b c}^{a} e^{b} f^{c}+\frac{a}{2 m^{2}} \epsilon_{b c}^{a} f^{b} f^{c}=0 .
$$

This equation can be solved for $f$ in terms of an infinite expansion

$$
f_{\mu \nu}=\sum_{n=0}^{\infty} \frac{1}{m^{2 n}} f_{\mu \nu}^{(n)}
$$

as follows:

$$
f_{\mu \nu}^{(n+1)}=-\frac{a}{2}\left(g_{\mu \rho} g_{\nu \sigma}-\frac{1}{2} g_{\mu \nu} g_{\rho \sigma}\right) \epsilon^{\rho \alpha \beta} \epsilon^{\sigma \gamma \delta} \sum_{k=0}^{n} f_{\alpha \gamma}^{(k)} f_{\beta \delta}^{(n-k)} .
$$

The starting value at order $m^{0}$ is $f_{\mu \nu}^{(0)}=-S_{\mu \nu}$. Having found the solution for $f_{\mu \nu}$, we can go to the metric formulation by plugging the solution of (5.4) back into the Lagrangian. The result can be written as

$$
\mathcal{L}=\frac{1}{2}\left\{\sigma R-2 \Lambda_{0}-\frac{2}{m^{2}}\left[\frac{2}{3} f_{\mu \nu} G^{\mu \nu}+\frac{1}{6}\left(f_{\mu \nu} f^{\mu \nu}-f^{2}\right)\right]\right\} .
$$

Here $f=f_{\mu \nu} g^{\mu \nu}$ and $f_{\mu \nu}$ is given in terms of the coefficients $f_{\mu \nu}^{(n)}$ in eq. (5.4). Explicitly, up to order $1 / m^{6}$ we have checked that the scalar ghost free combinations in (3.11) are recovered with the Cotton tensor set to zero. When $a=\sigma$ these are the same leading terms that occur in the expansion of the Born-Infeld extension of NMG [46, 47] — see [43] for a supersymmetric version. Our construction (5.5) compares nicely with an earlier proposal 
based on limits of a class of bimetric theories [34]. The linearized spectrum of this model includes only one massive graviton. Using the prescription explained in section 4.2 and appendix A we obtain the following expression for the central charge of the model:

$$
c=\frac{3 \ell}{2 G}\left[\sigma+\frac{1}{a}\left(-1+\sqrt{1+\frac{a}{m^{2} \ell^{2}}}\right)\right] .
$$

This coincides with the central charge computed in [47], when $a=\sigma$. As shown in [48, 49] for the choice of parameters where the central charge becomes zero, 3D Born-Infeld gravity arises as a suitable counterterm for gravity in $\mathrm{AdS}_{4}$.

Holographic $c$-theorem. Considering these higher-derivative models as toy models for exploring the role of higher-derivatives in holography, we observed that higher-derivative theories can accommodate terms involving $\nabla R$ which are introduced via the Cotton tensor in special combinations - see for instance (3.11) and (B.6). They are fully consistent with a holographic $c$-theorem. This feature can easily be generalized to higher dimensions by using the higher-dimensional Cotton and Schouten tensors:

$$
C_{\mu \nu \lambda}=(D-2)\left(\nabla_{\lambda} S_{\mu \nu}-\nabla_{\nu} S_{\mu \lambda}\right) \quad \text { and } \quad S_{\mu \nu}=\frac{1}{(D-2)}\left(R_{\mu \nu}-\frac{1}{2(D-1)} R g_{\mu \nu}\right) \text {. }
$$

In a sense, these tensors seem to be the right basis for studying higher-derivatives in the context of holography. Using the first order formulation, we particularly showed that the Einstein equations of higher-derivative theories in three dimensions, evaluated on the background (4.26), are always second order in derivatives.

Terms containing the Cotton tensor, do not contribute to the AdS one-point functions including the central charge. This covers a broader class of higher-derivative theories admitting a holographic $c$-theorem than the class of theories considered in [10, 25, 33], which only included higher-curvature terms containing $R^{n}$ tensors.

Since the presence of these higher-derivative terms leads to tachyons or ghosts, this observation confirms the conclusions that some 'unphysical' models with non-unitary operators still satisfy a holographic $c$-theorem [10].

Unitarity. The problem of non-unitarity is generic in higher-derivative models including the ones we consider here. The linear spectrum of these higher-derivative theories generically propagates massive spin- 2 modes. However, because of the higher-derivative nature of the theory, some of these massive modes are inherently unstable; this result is compatible with earlier higher-derivative extensions of general relativity, see for instance $[26,50]$. Due to the instability of the linearized massive modes, the applicability of these models may be limited to special, critical points in their parameter space; points where the linearized equations are only second order in derivatives and the massive modes disappear or points where they become massless and are replaced by log modes with logarithmic fall-off behavior towards the AdS boundary. The former points may indicate a linearization instability as it is unlikely that the ghost issue is cured at the non-linear level. The latter points may be of more interest as the dual CFT is expected to be a logarithmic CFT. Although LCFT's are non-unitary, they have a wide range of applications in statistical physics see for instance $[31,51]$ for recent reviews. 
The higher-derivative nature of the models we constructed in this work, exhibits the same "bulk-boundary unitarity problem" that is inherent to higher-derivative extensions of general relativity. This problem refers to the impossibility to obtain a positive boundary central charge (or black hole charge) for a region in parameter space that has well-behaved bulk spin-2 modes. Recently, a different CS-like theory for 3D gravity was introduced which resolves this problem. This model was called zwei-dreibein gravity (ZDG) [44]. ${ }^{10}$ The resolution of the bulk-boundary conflict stems from the fact that the parameter region of ZDG is large enough to include a well-behaved region as far as the sign of the central charge, the kinetic terms and the mass terms of the theory are concerned. This enhancement in parameters has the consequence that the ZDG action cannot be written in terms of (higher-derivatives of) a single metric. ${ }^{11}$ We expect that the bulk-boundary clash in the higher-derivative extensions of general relativity presented in this paper can similarly be resolved by considering a ZDG-like extension, generically called "viel-dreibein gravity", that involves more than 4 Lorentz vector-valued one-form fields [45].

Here we have only concentrated on AdS holography. These models can accommodate non-AdS spacetimes due to a large parameter space and might be unitary on these backgrounds. Specifically, there is a quantization preference in three dimensions in terms of unitarity. Unitary quantization of parity-odd theories seems to prefer asymptotically flat spacetimes, while AdS asymptotics are good for quantization of parity-even theories. Flat boundary conditions at null infinity in three dimensional gravity lead to a centrally extended $\mathrm{BMS}_{3}$ algebra as asymptotic symmetry algebra [56] — for a new derivation see [17]. The non-zero commutators are,

$$
\begin{aligned}
{\left[L_{m}, L_{n}\right] } & =(m-n) L_{m+n}+\frac{c_{L}}{12} m\left(m^{2}-1\right) \delta_{m+n, 0}, \\
{\left[L_{m}, M_{n}\right] } & =(m-n) M_{m+n}+\frac{c_{M}}{12} m\left(m^{2}-1\right) \delta_{m+n, 0},
\end{aligned}
$$

with Virasoro generators $L_{n}$ and supertranslations $M_{n}$. A unitary theory in flat space should have $c_{M}=0$. Then one is left with a single copy of the Virasoro algebra. This happens for free for all parity-odd gravity theories like conformal gravity $[17,57]$ — see [22] for a recent review. For all parity-odd higher-derivative gravity theories that we considered here the value of the AdS central charges are the same (4.30), therefore they lead to the same asymptotically flat symmetries with

$$
c_{L}=\frac{3}{\mu G}, \quad c_{M}=0 .
$$

This is a necessary condition for unitarity but not sufficient. The higher-derivative parityodd theories with flat boundary conditions might suffer from the same pathologies as in the parity-even ones with AdS boundary conditions; the massive modes can propagate negative norm states.

Another interesting development would be to construct a holographic dictionary for the class of Chern-Simons-like models with local bulk degrees of freedom. This could then

\footnotetext{
${ }^{10} \mathrm{ZDG}$ is a three dimensional, first-order formulation of bimetric gravity of [52] as first considered in [53].

${ }^{11}$ This is not true for the equations of motion, which feature an infinite expansion of higher-derivative terms [54]. For similar arguments in the metric formulation of the bimetric gravity of [52], see [55].
} 
directly be applied to the models we considered in this paper and to a variety of other CSlike models, such as ZDG and the recently introduced minimal massive gravity extension of TMG [36].

\section{Acknowledgments}

The authors wish to thank Mehmet Ozkan for helpful discussions. We thank Daniel Grumiller, Alasdair Routh, Paul Townsend and Thomas Zojer for their useful comments on the draft. H. R. A. and W. M. are supported by the Dutch stichting voor Fundamenteel Onderzoek der Materie (FOM).

\section{A Charges in Chern-Simons-like theories}

In this appendix we discuss the identification of the first class constraints of the CS-like theories and the corresponding boundary charges, which is of use in section 4 when we discuss the AdS holography for the higher-derivative gravity models we consider in this work.

\section{A.1 Canonical analysis}

The advantage of working with a Chern-Simons-like formulation of 3D higher-derivative gravity models is appreciated mostly when analyzing these theories in a Hamiltonian form and when computing their asymptotic symmetry algebra. All of the models we consider in this work belong to the following class of theories that are defined by a set of $N$ Lorentzvalued one-form fields with an action given by $[11]^{12}$

$$
S=\frac{1}{2 \kappa^{2}} \int_{\Sigma \times \mathbb{R}} g_{r s} a^{r} \cdot d a^{s}+\frac{1}{3} f_{r s t} a^{r} \cdot\left(a^{s} \times a^{t}\right) .
$$

Here $g_{r s}$ is a symmetric, constant and invertible metric on the flavor space which can be used to raise and lower flavor indices. The $f_{r s t}$ are totally symmetric structure constants; the theory is pure Chern-Simons when the expressions $\epsilon_{b c}^{a} f^{r}{ }_{s t}$ are the structure constants and $g_{r s} \eta_{a b}$ is a non-degenerate bilinear form of a Lie algebra.

The Hamiltonian formalism for this class of models was performed in [11, 12]. Here, we review some of the results of these references in order to fix the notation and derive the asymptotic symmetry algebra. After a space-time decomposition of the $N$ Lorentzvalued one-forms, the time components of the fields $a_{0}^{r}$ are Lagrange multipliers and $a_{i}^{r}$ are dynamical fields satisfying the following Poisson brackets,

$$
\left\{a_{i a}^{r}(x), a_{j b}^{s}(y)\right\}=\kappa^{2} \epsilon_{i j} g^{r s} \eta_{a b} \delta^{2}(x, y) .
$$

The Lagrange multipliers induce a set of $3 N$ primary constraints in the Hamiltonian analysis of the theory [12];

$$
\phi_{r}=\frac{1}{\kappa^{2}} \varepsilon^{i j}\left(g_{r s} \partial_{i} a_{j}^{s}+\frac{1}{2} f_{r s t}\left(a_{i}^{s} \times a_{j}^{t}\right)\right) .
$$

\footnotetext{
${ }^{12}$ Here we use a notation where wedge products between forms and Lorentz indices $a, b, \cdots$ are suppressed. The dots and crosses indicate contractions with $\eta_{a b}$ and $\epsilon_{a b c}$ respectively.
} 
It is convenient to define the "smeared" functions $\phi_{r}\left[\xi^{r}\right]$ associated to the constraint functions (A.3) by integrating them against a test function $\xi^{r}(x)$ as follows:

$$
\phi[\xi]=\sum_{r} \phi_{r}\left[\xi^{r}\right]=\int_{\Sigma} d^{2} x \xi^{r}(x) \cdot \phi_{r}(x)
$$

Here $\Sigma$ is a space-like hypersurface. In general, the variation of $\phi_{r}\left[\xi^{r}\right]$ may lead to non-zero boundary terms. Varying the expression (A.4) for $\phi[\xi]$ with respect to the fields $a_{i}{ }^{s}$ gives

$$
\delta \phi[\xi]=\int_{\Sigma} d^{2} x \xi^{r} \cdot \frac{\delta \phi_{r}}{\delta a_{i}^{s}} \cdot \delta a_{i}^{s}+\int_{\partial \Sigma} d x B[\xi, a, \delta a] .
$$

The boundary terms in this expression could lead to delta-function singularities in the Poisson brackets of the constraint functions. To remove these, we choose boundary conditions that make $B$ a total variation

$$
\int d x B[\xi, a, \delta a]=-\delta Q[\xi, a] .
$$

We can then define an improved set of constraint functions via

$$
\varphi[\xi]=\phi[\xi]+Q[\xi, a]
$$

These new functions will now have a well-defined variation without boundary terms. In our case, using eq. (A.3), we find

$$
\delta Q=-\frac{1}{\kappa^{2}} \int_{\partial \Sigma} d x^{i} g_{r s} \xi^{r} \cdot \delta a_{i}^{s}
$$

The Poisson brackets of the constraints were computed in $[11,12]$. They are given by

$$
\begin{aligned}
\{\varphi(\xi), \varphi(\eta)\}_{\text {P.B. }}= & \phi([\xi, \eta])+\frac{1}{\kappa^{2}} \int_{\Sigma} d^{2} x \xi_{a}^{r} \eta_{b}^{s} \mathcal{P}_{r s}^{a b} \\
& -\frac{1}{\kappa^{2}} \int_{\partial \Sigma} d x^{i} \xi^{r} \cdot\left(g_{r s} \partial_{i} \eta^{s}+f_{r s t}\left(a_{i}{ }^{s} \times \eta^{t}\right)\right),
\end{aligned}
$$

where we have defined

$$
\begin{aligned}
\mathcal{P}_{r s}^{a b} & =f_{q[r}^{t} f_{s] p t} \eta^{a b} \Delta^{p q}+2 f_{r[s}^{t} f_{q] p t}\left(V^{a b}\right)^{p q}, \\
V_{a b}^{p q} & =\varepsilon^{i j} a_{i a}^{p} a_{j b}^{q}, \quad \Delta^{p q}=\varepsilon^{i j} a_{i}^{p} \cdot a_{j}^{q}, \quad[\xi, \eta]^{t}=f_{r s}{ }^{t} \xi^{r} \times \eta^{s} .
\end{aligned}
$$

A detailed analysis of how to deal with the secondary constraints in this type of theories was presented in [12]. It suffices to state here that the secondary constraints derived in section 2.2 remove the $\Delta^{p q}$-term in the matrix $\mathcal{P}$ of Poisson brackets defined in eq. (A.10). For the purpose of this paper, it is sufficient to focus only on the algebra of constraint functions when adapting AdS (or Brown-Henneaux) boundary conditions. In the next section we will discuss the identification of the first class constraint functions. 


\section{A.2 First class constraint functions}

In contrast to the pure Chern-Simons gauge theories, not all constraint functions (A.3) in the Chern-Simons-like models are first class. In order to properly analyze the asymptotic symmetries, we should look at the algebra of first class constraint functions which generate the gauge symmetries. Hence, we first need to identify which (combination of) constraint functions generate the gauge symmetries of the theory.

The general CS-like theory defined by eq. (A.1) is manifestly diffeomorphism invariant. In addition, the specific CS-like models of our interest are also manifestly invariant under local Lorentz transformations. All models defined by the actions (2.7) and (2.8) contain a (dualized) spin-connection $\omega$, which only appears in terms of the dualized curvature twoform $R=\mathcal{D} \omega$ or via a Lorentz-Chern-Simons term in the action. Moreover, all derivatives of the other one-form fields $a^{r}$ appear as covariant derivatives $\mathcal{D} a^{r}$. Translated to components of the flavor space metric $g_{r s}$ and $f$-tensor $f_{r s t}$ this assumption is equivalent to the following statement

For every element of $g_{r s}$ there is a corresponding $f_{r s \omega}$ such that: $f_{r s \omega}=g_{r s}$.

Equipped with this assumption we should expect the CS-like models defined by (A.1) to have at least six gauge symmetries, corresponding to three diffeomorphism and three local Lorentz transformations.

To identify the constraint functions which generate these symmetries, it is instructive to look at the Poisson brackets of the gauge transformations with the dynamical components of the theory. In full generality (but omitting boundary terms at this point), they can be computed using the general formulas (A.3) and (A.4) with the following result:

$$
\left\{\phi[\xi], a_{i}^{r}\right\}=\partial_{i} \xi^{r}+f^{r}{ }_{s t} a_{i}^{s} \times \xi^{t} .
$$

From this result, we can deduce that a local Lorentz transformation with the gauge parameter $\tau$ is generated by the constraint function

$$
\phi_{\mathrm{LL}}[\tau] \equiv \phi_{\omega}\left[\xi^{\omega}\right] \quad \text { with } \quad \xi^{\omega}=\tau .
$$

With this identification we recover the usual transformation properties under local Lorentz transformations from (A.12):

$$
\begin{aligned}
& \delta_{\tau} \omega_{i}=\left\{\phi_{\mathrm{LL}}[\tau], \omega_{i}\right\}=\partial_{i} \tau+\omega_{i} \times \tau=\mathcal{D}_{i} \tau, \\
& \delta_{\tau} a_{i}^{r}=\left\{\phi_{\mathrm{LL}}[\tau], a_{i}^{r}\right\}=a_{i}^{r} \times \tau, \quad r \neq \omega,
\end{aligned}
$$

where we have used the fact that by the assumption (A.2) we may write $f^{r}{ }_{s \omega} \equiv g^{r p} f_{p s \omega}=$ $g^{r p} g_{p s}=\delta_{s}^{r}$.

In Chern-Simons gauge theories, diffeomorphisms are generated by an appropriate combination of constraint functions with parameters proportional to the fields [2], on-shell. The same is true for the general CS-like theory. Let us define

$$
\phi_{\text {diff }}[\zeta] \equiv \sum_{r} \phi_{r}\left[\xi^{r}\right] \quad \text { with } \quad \xi^{r}=a_{\mu}^{r} \zeta^{\mu}
$$


Then, by equation (A.12) we find that

$$
\delta_{\zeta} a_{i}^{r}=\left\{\phi_{\text {diff }}[\zeta], a_{i}^{r}\right\}=\mathcal{L}_{\zeta} a_{i}^{r}+\zeta^{\mu}\left(\partial_{i} a_{\mu}^{r}-\partial_{\mu} a_{i}^{r}+f^{r}{ }_{s t} a_{i}^{s} \times a_{\mu}^{t}\right)
$$

Here $\mathcal{L}_{\zeta}$ is the Lie derivative with respect to the vector field $\zeta^{\mu}$. The expressions in the parentheses are equivalent to the equations of motion of the general model (A.1) after a space-time decomposition. Hence, on-shell we have identified the constraint functions which give the correct transformation rules on the dynamical variables of the theory.

\section{A.3 Boundary charges}

To proceed with an analysis of the asymptotic symmetry group for AdS boundary conditions, we would like to write the algebra of first class constraint functions in a basis of mutually commuting $\mathrm{SL}(2, \mathbb{R})$ generators. This is possible on the AdS background as the generators of gauge symmetries should respect the isometries of the AdS vacuum solution. In all parity preserving models we can define such a basis as

$$
J_{ \pm}[\zeta]=\frac{1}{2}\left(\phi_{\mathrm{LL}}\left[e_{\mu} \zeta^{\mu}\right] \pm \ell \phi_{\mathrm{diff}}^{\prime}[\zeta]\right)
$$

where $\phi_{\text {diff }}^{\prime}[\zeta]=\phi_{\text {diff }}[\zeta]-\phi_{\mathrm{LL}}\left[\omega_{\mu} \zeta^{\mu}\right]$

At this point one should reinstate the boundary terms introduced in eq. (A.7), and investigate the Poisson bracket algebra of the generators (A.17) subject to asymptotically AdS (or Brown-Henneaux) boundary conditions. The improved differentiable generators are then,

$$
\mathcal{J}_{ \pm}[\zeta]=J_{ \pm}[\zeta]+Q_{ \pm}[\zeta]
$$

Let us first comment that, quite generally, by the fact that the auxiliary fields are symmetric (see eq. (2.10)), the gauge parameters for diffeomorphisms $\xi^{r}=a_{\mu}{ }^{r} \zeta^{\mu}$ satisfy

$$
e_{\mu} \cdot \xi^{f_{I}}=e_{\mu} \cdot f_{I \nu} \zeta^{\nu}=f_{I \mu} \cdot e_{\nu} \zeta^{\nu}=f_{I \mu} \cdot \xi^{e}, \quad e_{\mu} \cdot \xi^{h_{I}}=h_{I \mu} \cdot \xi^{e}
$$

Moreover, on the AdS background, since the auxiliary fields $f_{I}$ are all proportional to the AdS dreibein and the auxiliary fields $h_{I}$ vanish, we have

$$
\xi^{f_{I}}=\bar{f}_{I} \xi^{e}, \quad \xi^{h_{I}}=0,
$$

where $\bar{f}_{I}$ is the constant background value of the auxiliary fields, i.e. $\bar{f}_{I}{ }^{a}=\bar{f}_{I} \bar{e}^{a}$ on the AdS background. The values for $\bar{f}_{1}$ and $\bar{f}_{2}$ can be read from eq. (3.14). This allows us to express all the gauge parameters occurring in $\phi_{\text {diff }}^{\prime}[\zeta]$ in terms of $\xi^{e}=e_{\mu} \zeta^{\mu}$. Using these relations, we can compute the variation of the boundary terms in (A.18) from (A.8);

$$
\begin{aligned}
\delta Q_{ \pm}\left[\xi_{ \pm}^{e}\right] & =-\frac{1}{2 \kappa^{2}} \int_{\partial \Sigma} d x^{i}\left(g_{\omega s} \xi_{ \pm}^{e} \cdot \delta a_{i}^{s} \pm \ell g_{r s} \xi_{ \pm}^{r} \cdot \delta a_{i}^{s}\right) \\
& =-\frac{1}{2 \kappa^{2}} \int_{\partial \Sigma} d x^{i} \xi_{ \pm}^{e} \cdot\left[g_{\omega s} \delta a_{i}^{s}+\cdots \pm \ell\left(g_{e s}+\bar{f}_{1} g_{f_{1} s}+\bar{f}_{2} g_{f_{2} s}\right) \delta a_{i}^{s}\right]
\end{aligned}
$$


where in the first line the sum over $r$ does not include $\omega$ and in the second line we have used eq. (A.20). In general, after plugging in the explicit flavor space metric and AdS background values of the fields, the result may be written as

$$
\delta Q_{ \pm}\left[\xi^{ \pm}\right]=\frac{\hat{k}}{2 \pi} \int_{\partial \Sigma} d x^{i} \xi_{a}^{ \pm}\left(\delta \omega_{i}^{a} \pm \frac{1}{\ell} \delta e_{i}^{a}\right) .
$$

where $\hat{k}$ is an effective dimensionless coupling determined by the elements of $g_{r s}$ and the $\bar{f}_{I}$ 's. We have also distinguished the gauge parameters for the left and right moving sectors explicitly;

$$
\xi^{ \pm}= \pm \frac{1}{2} \xi_{ \pm}^{e}
$$

After imposing suitable boundary conditions and restricting $\xi^{ \pm}$to the set of symmetry transformations which preserve these boundary conditions it is possible to integrate this expression to obtain the global conserved charges of the theory. This is done explicitly in section 4.2 for Brown-Henneaux boundary conditions. The effective coupling $\hat{k}$ is related to the central charge as $c=\tilde{c}=6 \hat{k}$ for the parity-even models and $c=-\tilde{c}=6 \hat{k}$ for the parity-odd models.

As an example of these general considerations, let us compute the effective coupling $\hat{k}$ for ENMG explicitly. The parity-odd theory defined by $S_{3}$ will be treated in full detail in appendix B. Using the above relations and the specific values of $g_{r s}$ and $f_{r s t}$ for ENMG, which may be read off from eq. (3.1), we find that the variation of the conserved charges are given by (A.22) with:

$$
\hat{k}^{\mathrm{ENMG}}=\frac{\ell}{4 G}\left(\sigma+\frac{1}{2 m^{2} \ell^{2}}-\frac{a}{8 m^{4} \ell^{4}}\right) .
$$

\section{B Extended gravitational Chern-Simons term}

In this appendix we consider the class of parity-odd theories given in eq. (2.9). Applying the same procedure as in the parity-even case the following expression for the Lagrangian 3 -form that describes the leading five derivative extension in the odd sector can be derived:

$$
\begin{aligned}
L_{3}=\frac{1}{2 \mu} \omega_{a}\left(d \omega^{a}+\frac{1}{3} \epsilon_{a b c} \omega^{b} \omega^{c}\right)+\frac{1}{\mu^{3}} & e_{a} \mathcal{D} f_{2}{ }^{a}+h_{1 a}\left(R^{a}+\epsilon^{a b c} e_{b} f_{1 c}\right) \\
& \left.+\frac{\alpha}{2} f_{1 a} \mathcal{D} f_{1}{ }^{a}\right] .
\end{aligned}
$$

Here, $\alpha$ is an arbitrary dimensionless parameter. The equations of motion for this Lagrangian, obtained by varying with respect to $f_{2}{ }^{a}, h_{1}{ }^{a}, f_{1}{ }^{a}, \omega^{a}$ and $e^{a}$, respectively, are given by

$$
\begin{aligned}
& \mathcal{D} e^{a}=0 \\
& R^{a}+\epsilon^{a}{ }_{b c} e^{b} f_{1}{ }^{c}=0, \\
& \alpha \mathcal{D} f_{1}{ }^{a}+\epsilon^{a}{ }_{b c} e^{b} h_{1}{ }^{c}=0, \\
& \mathcal{D} h_{1}{ }^{a}+\frac{1}{2} \epsilon^{a}{ }_{b c}\left(\alpha f_{1}{ }^{b} f_{1}{ }^{c}+2 \epsilon^{a}{ }_{b c} e^{b} f_{2}{ }^{c}-2 \mu^{2} \sigma \epsilon^{a}{ }_{b c} e^{b} f_{1}{ }^{c}\right)=0, \\
& \mathcal{D} f_{2}{ }^{a}+\epsilon^{a}{ }_{b c} f_{1}{ }^{b} h_{1}{ }^{c}=0 .
\end{aligned}
$$


Upon acting on the equations of motion with an exterior derivative and performing some algebra, one can derive that the auxiliary fields are symmetric

$$
f_{1[\mu \nu]}=h_{1[\mu \nu]}=f_{2[\mu \nu]}=0 .
$$

We can solve them in turns of derivatives of the dreibein. Explicitly, one finds the following expressions:

$$
f_{1 \mu \nu}=-S_{\mu \nu}, \quad h_{1 \mu \nu}=\alpha C_{\mu \nu}, \quad f_{2 \mu \nu}=-\alpha D_{\mu \nu}+\alpha\left(P_{\mu \nu}-\frac{1}{4} P g_{\mu \nu}\right)-\mu^{2} S_{\mu \nu},
$$

where $P_{\mu \nu}$ and $D_{\mu \nu}$ are defined in (3.6). Substituting these solutions back into the action leads to the following extended Lagrangian density:

$$
\begin{aligned}
\mathcal{L}_{3} & =\mathcal{L}_{1}+\frac{\alpha}{\mu^{3}} S_{\mu \nu} C^{\mu \nu} \\
& =\frac{1}{\mu} \epsilon^{\mu \nu \lambda}\left\{\Gamma_{\mu \sigma}^{\rho} \partial_{\nu} \Gamma_{\lambda \rho}^{\sigma}+\frac{2}{3} \Gamma_{\mu \sigma}^{\rho} \Gamma_{\nu \tau}^{\sigma} \Gamma_{\lambda \rho}^{\tau}+\frac{\alpha}{\mu^{2}} R_{\mu}{ }^{\sigma} \nabla_{\nu} R_{\sigma \lambda}\right\} .
\end{aligned}
$$

Applying the same procedure one step further we find the following seven derivative Lagrangian density:

$$
\mathcal{L}_{5}=\mathcal{L}_{3}+\frac{1}{\mu^{5}}\left\{\beta_{1} P_{\mu \nu} C^{\mu \nu}+\beta_{2} D_{\mu \nu} C^{\mu \nu}\right\}
$$

where $\beta_{1}$ and $\beta_{2}$ are two dimensionless parameters and $P_{\mu \nu}$ and $D_{\mu \nu}$ are defined in (3.6).

\section{B.1 Linearization}

To obtain the quadratic Lagrangian we substitute the fluctuations (3.14) into the action (B.1) with $m \rightarrow \mu$ and $a \rightarrow \alpha$. After making the field redefinitions

$$
k_{2} \rightarrow k_{2}+\left(\mu^{2}+\frac{\alpha \Lambda}{2}\right) k_{1}-\frac{\alpha \Lambda^{2}}{8} k \quad \text { and } \quad k_{1} \rightarrow k_{1}-\frac{\Lambda}{2} k,
$$

we obtain the following quadratic Lagrangian for $S_{3}$ :

$$
\begin{aligned}
L_{3}^{(2)}=L_{1}^{(2)}+\frac{1}{\mu^{3}}\{ & k_{2 a}\left(\overline{\mathcal{D}} k^{a}+\epsilon^{a}{ }_{b c} \bar{e}^{b} v^{c}\right)+v_{1 a}\left(\overline{\mathcal{D}} v^{a}-\Lambda \epsilon^{a}{ }_{b c} \bar{e}^{b} k^{c}+\epsilon^{a}{ }_{b c} \bar{e}^{b} k_{1}^{c}\right) \\
& \left.+\frac{\alpha}{2} k_{1} \overline{\mathcal{D}} k_{1}\right\} .
\end{aligned}
$$

Upon eliminating the auxiliary fields by their equations of motion, we find the five derivative Lagrangian density

$$
\mathcal{L}_{3}^{(2)}=-\frac{1}{\mu} \epsilon^{\mu \alpha}{ }_{\rho} \bar{\nabla}_{\alpha} k^{\rho \nu} \mathcal{G}_{\mu \nu}(k)+\frac{\alpha}{\mu^{3}} \epsilon^{\mu \alpha}{ }_{\rho} \bar{\nabla}_{\alpha} \mathcal{G}^{\rho \nu}(k) \mathcal{G}_{\mu \nu}(k),
$$

where $\mathcal{G}_{\mu \nu}$ is defined in (3.19). The linearized equations of motion for the action corresponding to this Lagrangian density can be written as:

$$
\left(\mathcal{D}^{0} \mathcal{D}^{M} \tilde{\mathcal{D}}^{M} \mathcal{D}^{L} \mathcal{D}^{R} k\right)_{\mu \nu}=0,
$$


where $\left(\mathcal{D}^{L / R}\right)_{\mu}^{\rho}=\delta_{\mu}^{\rho} \pm \ell \epsilon_{\mu}{ }^{\alpha \rho} \bar{\nabla}_{\alpha}$ and the differential operator $\mathcal{D}^{0}$ is defined in (3.20) while $\mathcal{D}^{M}$ and $\tilde{\mathcal{D}}^{M}$ are defined as in (4.8) with the mass parameter $M$ given by:

$$
M=\sqrt{\mathcal{M}^{2}-\Lambda}=\sqrt{\frac{1}{\ell^{2}}-\frac{\mu^{2}}{\alpha}} .
$$

The linear theory hence describes a partially massless mode, and two helicity- \pm 2 massive modes, with a Fierz-Pauli mass $\mathcal{M}^{2}=-\mu^{2} / \alpha$. Note that the conformal symmetry is broken due to the additional interaction term. The theory hence propagates three degrees of freedom, corresponding to the two helicity states of the massive mode and the partially massless mode.

In accordance with what is expected from the linear spectrum, it is possible to diagonalize the quadratic Lagrangian. After making the appropriate field redefinitions the Lagrangian density (B.8) can be written as:

$$
\begin{aligned}
L_{3}^{(2)}= & \frac{1}{\mu}\left(k_{L}^{a} \overline{\mathcal{D}} k_{L a}+\frac{1}{\ell} \epsilon_{a b c} \bar{e}^{a} k_{L}^{b} k_{L}^{c}\right)+\frac{1}{\mu}\left(k_{R}^{a} \overline{\mathcal{D}} k_{R a}-\frac{1}{\ell} \epsilon_{a b c} \bar{e}^{a} k_{R}{ }^{b} k_{R}^{c}\right) \\
& +\frac{\left(\alpha-\ell^{2} \mu^{2}\right)}{2 \mu} k_{0}^{a} \overline{\mathcal{D}} k_{0 a}-\frac{1}{\mu}\left(k_{M_{+}}^{a} \overline{\mathcal{D}} k_{M_{+} a}+M \epsilon_{a b c} \bar{e}^{a} k_{M_{+}}^{b} k_{M_{+}}^{c}\right) \\
& -\frac{1}{\mu}\left(k_{M_{-}}^{a} \overline{\mathcal{D}} k_{M_{-} a}-M \epsilon_{a b c} \bar{e}^{a} k_{M_{-}}^{b} k_{M_{-}}^{c}\right),
\end{aligned}
$$

where we have assumed that $\mu^{2} \ell^{2} \neq \alpha$. The modes indexed by $L / R / 0$ or $M_{+/-}$are annihilated by $\mathcal{D}^{L / R / 0}$ and $\mathcal{D}^{M} / \tilde{\mathcal{D}}^{M}$ respectively. The point $\alpha=\ell^{2} \mu^{2}$ corresponds to a special case in the linear spectrum where the massive modes become partially massless and degenerate with the partially massless mode $k_{0}$. Note that there is no (finite) parameter choice possible where the massive mode degenerates with the massless mode and the massive and the massless sectors come with opposite signs.

\section{B.2 Central charge}

The extended Lagrangian (B.1) fits the general model (A.1) with flavor space metric and structure constants given by

$$
\begin{aligned}
& g_{\omega \omega}=\frac{1}{\mu}, \quad g_{e f_{2}}=g_{\omega h_{1}}=\frac{1}{\mu^{3}}, \quad g_{f_{1} f_{1}}=\frac{\alpha}{\mu^{3}}, \\
& f_{\omega \omega \omega}=\frac{1}{\mu}, \quad f_{\omega e f_{2}}=f_{\omega \omega h_{1}}=f_{e f_{1} h_{1}}=\frac{1}{\mu^{3}}, \quad f_{\omega f_{1} f_{1}}=\frac{\alpha}{\mu^{3}} .
\end{aligned}
$$

The matrix of Poisson brackets (A.10) in the flavor space basis $\left(\omega, e, f_{1}, h_{1}, f_{2}\right)$ is given by

$$
\mathcal{P}=\left(\begin{array}{ll}
0 & 0 \\
0 & Q
\end{array}\right),
$$

with $Q$ given by

$$
Q=\frac{1}{\mu^{3}}\left(\begin{array}{cccc}
\mu^{2} V_{a b}^{f_{1} f_{1}}-\frac{1}{\alpha} V_{a b}^{h_{1} h_{1}}-2 V_{[a b]}^{f_{1} f_{2}}-\mu^{2} V_{a b}^{f_{1} e}+V_{a b}^{f_{2} e} & \frac{1}{\alpha} V_{a b}^{h_{1} e} & V_{a b}^{f_{1} e} \\
-\mu^{2} V_{a b}^{e f_{1}}+V_{a b}^{e f_{2}} & \mu^{2} V_{a b}^{e e} & 0 & -V_{a b}^{e e} \\
\frac{1}{\alpha} V_{a b}^{e h_{1}} & 0 & -\frac{1}{\alpha} V_{a b}^{e e} & 0 \\
V_{a b}^{e f_{1}} & -V_{a b}^{e e} & 0 & 0
\end{array}\right) .
$$


From (B.15) it is clear that $\phi_{\mathrm{LL}}[\chi]$ defined by (A.13) is first class. To show that the brackets of $\phi_{\text {diff }}[\zeta]$ vanish, we may use that, by virtue of

$$
e_{\left[\mu^{a}\right.}^{a} f_{1 \nu] a}=e_{[\mu}^{a} h_{1 \nu] a}=e_{\left[\mu^{a}\right.}^{a} f_{2 \nu] a}=0,
$$

the gauge parameters $\xi_{a}^{r}=a_{\mu a}^{r} \zeta^{\mu}$ satisfy

$$
e_{i}{ }^{a} \xi_{a}^{f_{1}}=f_{1 i}{ }^{a} \xi_{a}^{e}, \quad e_{i}{ }^{a} \xi_{a}^{h_{1}}=h_{1 i}{ }^{a} \xi_{a}^{e}, \quad e_{i}{ }^{a} \xi_{a}^{f_{2}}=f_{2 i}{ }^{a} \xi_{a}^{e} .
$$

Using these identities, explicit computation shows that $\phi_{\text {diff }}[\zeta]$ as defined in (A.15) has weakly vanishing brackets with all other primary constraint functions. It is also possible to show that the Poisson brackets of $\phi_{\mathrm{LL}}[\chi]$ and $\phi_{\text {diff }}[\zeta]$ with the secondary constraints vanish on the AdS vacuum. This is sufficient to identify them as the generators of the gauge symmetries at the AdS boundary, since close to the AdS boundary, we may use the background values for the fields. Then, it becomes possible to split the first class constraint functions into a set of mutually commuting constraints $J_{ \pm}$defined by eq. (A.17). From the background values of the fields we derive that

$$
\xi_{a}^{f_{1}}=\frac{1}{2 \ell^{2}} \xi_{a}^{e}, \quad \xi_{a}^{h_{1}}=0, \quad \xi_{a}^{f_{2}}=\frac{1}{2 \ell^{2}}\left(\mu^{2}-\frac{\alpha}{4 \ell^{2}}\right) \xi_{a}^{e} .
$$

Upon using the AdS background identities (B.19) in the expression for the boundary charges (A.21), we find that

$$
\delta Q_{3}^{ \pm}\left[\xi^{ \pm}\right]= \pm \frac{1}{8 \pi \mu G} \int_{\partial \Sigma} d x^{i} \xi_{a}^{ \pm}\left(\delta \omega_{i}^{a} \pm \frac{1}{\ell} \delta e_{i}^{a}\right),
$$

where we have reinstated the overall factor of $\kappa^{2}=8 \pi G$. Following the asymptotic analysis of section 4.2 , this leads to a central charge given by,

$$
c_{3}=-\tilde{c}_{3}=\frac{3}{2 \mu G} .
$$

We observe that the result does not depend on the new coupling constant $\alpha$. This is consistent with the result of section 4.4 which states that the central charge in the odd sector is universal.

Open Access. This article is distributed under the terms of the Creative Commons Attribution License (CC-BY 4.0), which permits any use, distribution and reproduction in any medium, provided the original author(s) and source are credited.

\section{References}

[1] A. Achucarro and P.K. Townsend, A Chern-Simons Action for Three-Dimensional anti-de Sitter Supergravity Theories, Phys. Lett. B 180 (1986) 89 [InSPIRE].

[2] E. Witten, (2+1)-Dimensional Gravity as an Exactly Soluble System, Nucl. Phys. B 311 (1988) 46 [InSPIRE].

[3] M. Bañados, C. Teitelboim and J. Zanelli, The Black hole in three-dimensional space-time, Phys. Rev. Lett. 69 (1992) 1849 [hep-th/9204099] [INSPIRE]. 
[4] M. Bañados, M. Henneaux, C. Teitelboim and J. Zanelli, Geometry of the (2+1) black hole, Phys. Rev. D 48 (1993) 1506 [gr-qc/9302012] [inSPIRE].

[5] J.D. Brown and M. Henneaux, Central Charges in the Canonical Realization of Asymptotic Symmetries: An Example from Three-Dimensional Gravity, Commun. Math. Phys. 104 (1986) 207 [INSPIRE].

[6] S. Deser, R. Jackiw and S. Templeton, Topologically Massive Gauge Theories, Annals Phys. 140 (1982) 372 [Erratum ibid. 185 (1988) 406] [INSPIRE].

[7] E.A. Bergshoeff, O. Hohm and P.K. Townsend, Massive Gravity in Three Dimensions, Phys. Rev. Lett. 102 (2009) 201301 [arXiv:0901.1766] [inSPIRE].

[8] A.B. Zamolodchikov, Irreversibility of the Flux of the Renormalization Group in a $2 D$ Field Theory, JETP Lett. 43 (1986) 730 [INSPIRE].

[9] D.Z. Freedman, S.S. Gubser, K. Pilch and N.P. Warner, Renormalization group flows from holography supersymmetry and a c theorem, Adv. Theor. Math. Phys. 3 (1999) 363 [hep-th/9904017] [INSPIRE].

[10] R.C. Myers and A. Sinha, Holographic c-theorems in arbitrary dimensions, JHEP 01 (2011) 125 [arXiv:1011.5819] [INSPIRE].

[11] O. Hohm, A. Routh, P.K. Townsend and B. Zhang, On the Hamiltonian form of $3 D$ massive gravity, Phys. Rev. D 86 (2012) 084035 [arXiv: 1208.0038] [INSPIRE].

[12] E.A. Bergshoeff, O. Hohm, W. Merbis, A.J. Routh and P.K. Townsend, The Hamiltonian Form of Three-Dimensional Chern-Simons-like Gravity Models, arXiv:1402.1688 [INSPIRE].

[13] E. Witten, Three-Dimensional Gravity Revisited, arXiv:0706.3359 [INSPIRE].

[14] H. Afshar, B. Cvetkovic, S. Ertl, D. Grumiller and N. Johansson, Holograms of Conformal Chern-Simons Gravity, Phys. Rev. D 84 (2011) 041502 [arXiv: 1106.6299] [InSPIRE].

[15] H. Afshar, B. Cvetkovic, S. Ertl, D. Grumiller and N. Johansson, Conformal Chern-Simons holography - lock, stock and barrel, Phys. Rev. D 85 (2012) 064033 [arXiv:1110.5644] [INSPIRE].

[16] J.H. Horne and E. Witten, Conformal Gravity in Three-dimensions as a Gauge Theory, Phys. Rev. Lett. 62 (1989) 501 [INSPIRE].

[17] H.R. Afshar, Flat/AdS boundary conditions in three dimensional conformal gravity, JHEP 10 (2013) 027 [arXiv: 1307.4855] [INSPIRE].

[18] E.A. Bergshoeff, O. Hohm and P.K. Townsend, More on Massive 3D Gravity, Phys. Rev. D 79 (2009) 124042 [arXiv:0905.1259] [INSPIRE].

[19] S. Carlip, The Constraint Algebra of Topologically Massive AdS Gravity, JHEP 10 (2008) 078 [arXiv:0807.4152] [INSPIRE].

[20] M. Blagojevic and B. Cvetkovic, Canonical structure of topologically massive gravity with a cosmological constant, JHEP 05 (2009) 073 [arXiv: 0812.4742] [INSPIRE].

[21] M. Blagojevic and B. Cvetkovic, Hamiltonian analysis of BHT massive gravity, JHEP 01 (2011) 082 [arXiv: 1010.2596] [INSPIRE].

[22] H. Afshar, A. Bagchi, S. Detournay, D. Grumiller, S. Prohazka, and M. Riegler, Holographic Chern-Simons Theories, arXiv:1404.1919 [INSPIRE]. 
[23] C. de Rham, G. Gabadadze, D. Pirtskhalava, A.J. Tolley and I. Yavin, Nonlinear Dynamics of 3D Massive Gravity, JHEP 06 (2011) 028 [arXiv:1103.1351] [INSPIRE].

[24] D.G. Boulware and S. Deser, Can gravitation have a finite range?, Phys. Rev. D 6 (1972) 3368 [inSPIRE].

[25] A. Sinha, On the new massive gravity and AdS/CFT, JHEP 06 (2010) 061 [arXiv: 1003.0683] [INSPIRE].

[26] E.A. Bergshoeff, S. de Haan, W. Merbis, J. Rosseel and T. Zojer, On Three-Dimensional Tricritical Gravity, Phys. Rev. D 86 (2012) 064037 [arXiv: 1206.3089] [InSPIRE].

[27] D. Grumiller and N. Johansson, Instability in cosmological topologically massive gravity at the chiral point, JHEP 07 (2008) 134 [arXiv:0805.2610] [INSPIRE].

[28] D. Grumiller and N. Johansson, Consistent boundary conditions for cosmological topologically massive gravity at the chiral point, Int. J. Mod. Phys. D 17 (2009) 2367 [arXiv:0808.2575] [INSPIRE].

[29] M. Henneaux, C. Martinez and R. Troncoso, Asymptotically anti-de Sitter spacetimes in topologically massive gravity, Phys. Rev. D 79 (2009) 081502 [arXiv:0901.2874] [INSPIRE].

[30] A. Maloney, W. Song and A. Strominger, Chiral Gravity, Log Gravity and Extremal CFT, Phys. Rev. D 81 (2010) 064007 [arXiv:0903.4573] [INSPIRE].

[31] D. Grumiller, W. Riedler, J. Rosseel and T. Zojer, Holographic applications of logarithmic conformal field theories, J. Phys. A 46 (2013) 494002 [arXiv:1302.0280] [InSPIRE].

[32] S. Deser and A. Waldron, Gauge invariances and phases of massive higher spins in $(A) d S$, Phys. Rev. Lett. 87 (2001) 031601 [hep-th/0102166] [INSPIRE].

[33] M.F. Paulos, New massive gravity extended with an arbitrary number of curvature corrections, Phys. Rev. D 82 (2010) 084042 [arXiv: 1005.1646] [INSPIRE].

[34] M.F. Paulos and A.J. Tolley, Massive Gravity Theories and limits of Ghost-free Bigravity models, JHEP 09 (2012) 002 [arXiv:1203.4268] [INSPIRE].

[35] E.A. Bergshoeff, O. Hohm, J. Rosseel, E. Sezgin and P.K. Townsend, On Critical Massive (Super)Gravity in adS3, J. Phys. Conf. Ser. 314 (2011) 012009 [arXiv:1011.1153] [INSPIRE].

[36] E. Bergshoeff, O. Hohm, W. Merbis, A.J. Routh and P.K. Townsend, Minimal Massive 3D Gravity, Class. Quant. Grav. 31 (2014) 145008 [arXiv:1404.2867] [INSPIRE].

[37] M. Bañados, Global charges in Chern-Simons field theory and the (2+1) black hole, Phys. Rev. D 52 (1996) 5816 [hep-th/9405171] [INSPIRE].

[38] W. Li, W. Song and A. Strominger, Chiral Gravity in Three Dimensions, JHEP 04 (2008) 082 [arXiv: 0801.4566] [INSPIRE].

[39] O. Coussaert, M. Henneaux and P. van Driel, The Asymptotic dynamics of three-dimensional Einstein gravity with a negative cosmological constant, Class. Quant. Grav. 12 (1995) 2961 [gr-qc/9506019] [INSPIRE].

[40] D. Grumiller, N. Johansson and T. Zojer, Short-cut to new anomalies in gravity duals to logarithmic conformal field theories, JHEP 01 (2011) 090 [arXiv: 1010.4449] [INSPIRE].

[41] D. Grumiller and O. Hohm, AdS $S_{3} / L C F T$ (2): Correlators in New Massive Gravity, Phys. Lett. B 686 (2010) 264 [arXiv:0911.4274] [INSPIRE]. 
[42] M. Alishahiha and A. Naseh, Holographic renormalization of new massive gravity, Phys. Rev. D 82 (2010) 104043 [arXiv: 1005.1544] [InSPIRE].

[43] E. Bergshoeff and M. Ozkan, 3D Born-Infeld Gravity and Supersymmetry, arXiv: 1405.6212 [INSPIRE].

[44] E.A. Bergshoeff, S. de Haan, O. Hohm, W. Merbis and P.K. Townsend, Zwei-Dreibein Gravity: A Two-Frame-Field Model of 3D Massive Gravity, Phys. Rev. Lett. 111 (2013) 111102 [arXiv:1307.2774] [INSPIRE].

[45] H.R. Afshar, E.A. Bergshoeff and W. Merbis, Drei-Dreibein Gravity and beyond, To appear (2014).

[46] I. Gullu, T.C. Sisman and B. Tekin, Born-Infeld extension of new massive gravity, Class. Quant. Grav. 27 (2010) 162001 [arXiv:1003.3935] [INSPIRE].

[47] I. Gullu, T.C. Sisman and B. Tekin, c-functions in the Born-Infeld extended New Massive Gravity, Phys. Rev. D 82 (2010) 024032 [arXiv: 1005.3214] [InSPIRE].

[48] D.P. Jatkar and A. Sinha, New Massive Gravity and AdS $S_{4}$ counterterms, Phys. Rev. Lett. 106 (2011) 171601 [arXiv:1101.4746] [INSPIRE].

[49] K. Sen, A. Sinha and N.V. Suryanarayana, Counterterms, critical gravity and holography, Phys. Rev. D 85 (2012) 124017 [arXiv:1201.1288] [INSPIRE].

[50] T. Nutma, Polycritical Gravities, Phys. Rev. D 85 (2012) 124040 [arXiv:1203.5338] [INSPIRE].

[51] J. Cardy, Logarithmic conformal field theories as limits of ordinary CFTs and some physical applications, J. Phys. A 46 (2013) 494001 [arXiv:1302.4279] [inSPIRE].

[52] S.F. Hassan and R.A. Rosen, Bimetric Gravity from Ghost-free Massive Gravity, JHEP 02 (2012) 126 [arXiv:1109.3515] [INSPIRE].

[53] K. Hinterbichler and R.A. Rosen, Interacting Spin-2 Fields, JHEP 07 (2012) 047 [arXiv: 1203.5783] [INSPIRE].

[54] E.A. Bergshoeff, A.F. Goya, W. Merbis and J. Rosseel, Logarithmic AdS Waves and Zwei-Dreibein Gravity, JHEP 04 (2014) 012 [arXiv:1401.5386] [INSPIRE].

[55] S.F. Hassan, A. Schmidt-May and M. von Strauss, Higher Derivative Gravity and Conformal Gravity From Bimetric and Partially Massless Bimetric Theory, arXiv:1303.6940 [INSPIRE].

[56] G. Barnich and G. Compere, Classical central extension for asymptotic symmetries at null infinity in three spacetime dimensions, Class. Quant. Grav. 24 (2007) F15 [gr-qc/0610130] [INSPIRE].

[57] A. Bagchi, S. Detournay and D. Grumiller, Flat-Space Chiral Gravity, Phys. Rev. Lett. 109 (2012) 151301 [arXiv:1208.1658] [INSPIRE]. 